\title{
Lattice Boltzmann modeling of interfacial gravity waves
}

\author{
J. M. Buick and C. A. Greated \\ Department of Physics and Astronomy, The University of Edinburgh, The Kings Buildings, Mayfield Road, \\ Edinburgh, EH9 3JZ, United Kingdom
}

(Received 22 August 1997; accepted 20 February 1998)

\begin{abstract}
A novel numerical technique for simulating interfacial gravity waves is considered. The model comprises an immiscible, binary fluid lattice Boltzmann model incorporating a gravitational interaction and simulates two immiscible, viscous fluids of different densities with a sharp interface between them. The model is described and the accuracy of the technique is considered. Simulation results are presented and the wave velocities, the oscillation frequency and the damping rate are found and compared to theory. A good comparison is found suggesting that the lattice Boltzmann approach is indeed accurately mimicking the wave dynamics. (C) 1998 American Institute of
\end{abstract} Physics. [S1070-6631(98)01106-4]

\section{INTRODUCTION}

Internal gravity waves at a density change can occur in coastal waters, ${ }^{1-3}$ fjords, ${ }^{4}$ lakes ${ }^{5,6}$ and in the atmosphere. ${ }^{7-9}$ The first record of the motion of internal waves was made during Nansen's expedition to the Arctic Ocean between 1893 and 1896 aboard the FRAM. ${ }^{10}$ One observation was the "dead-water" phenomenon where the resistance of the ship traveling through the water would increase without any visible outlay of energy; such as the production of surface waves. A study of this was performed by Ekman ${ }^{11}$ who concluded that the dead-water phenomenon was caused by the production of internal gravity waves. Now, over a century later, there is still a great deal of interest in internal waves and related topics, ${ }^{12}$ since a full understanding of their motion is important in, for example, the design of oil drilling structures $^{13}$ and under water bridges. ${ }^{14}$

Here we consider two-dimensional waves in a two-layer fluid. Internal waves of this form are called interfacial waves and are typical of the internal waves between two fluids of different densities which occur, for example, when a layer of fresh water from a river flows into salt water; and which are a good approximation to waves in a fluid where the density changes rapidly, such as at a steep thermocline. Early theoretical studies of this problem include the prediction of the wave frequency and damping rate when one or both of the fluids are infinite ${ }^{15}$ and when both fluids are finite ${ }^{16}$ both in the linear limit of small wave amplitudes. Linear internal waves have also been studied by Thorpe ${ }^{17}$ who examined linear standing waves as well as considering standing waves with a larger amplitude. A further experimental study of progressive nonlinear waves ${ }^{18}$ was found to compare well with a third-order Stokes expansion. Higher-order Stokes expansions have also been considered. ${ }^{19}$ An experimental and theoretical investigation into long progressive internal waves over a slope $\mathrm{e}^{20}$ has shown shearing and breaking effects. Weakly nonlinear progressive and standing waves have also been studied ${ }^{21}$ and the existence of mixing waves which are formed by the combination of standing and progressive waves was proved for bounded fluids. Mixing waves have also been observed in infinite fluids. ${ }^{22}$ Koop et al. ${ }^{23}$ and more recently Michallet et al. ${ }^{24}$ have made experimental studies of interfacial solitary waves between immiscible fluids with different specific gravities. These were compared favorably with nonlinear wave theories. Numerical studies have concentrated on incompressible, inviscid, irrotational fluids. Vanden-Broeck ${ }^{25}$ studied gravity-capillary interfacial waves between two semi-infinite immiscible fluids of different densities by considering an integro-differential equation and converting it into a set of nonlinear algebraic equations which were solved by Newton's method. A similar approach was applied ${ }^{26}$ to simulate overhanging gravity waves. Steep interfacial waves have also been considere ${ }^{27-29}$ between inviscid fluids of different depths. All these numerical studies considered both fluids to be irrotational. Numerical studies have been performed ${ }^{30,31}$ in which the upper fluid is allowed to have a constant nonzero vorticity while the lower fluid is irrotational. As far as the authors are aware the numerical simulation of interfacial waves between two fully viscous fluids has not been considered, despite the evidence that the decay of internal waves is important. ${ }^{32}$ Here, using the lattice Boltzmann model we simulated interfacial waves between two immiscible fluids which have different densities and a variable viscosity. The technique used here is a new and potentially very powerful approach to interfacial wave modeling. In order to test the model and show that it is correctly simulating wave behavior, linear waves are considered where theoretical expressions for the motion are well established. A discussion of the application of the lattice Boltzmann model to more extreme waves and other problems of interest is given in Sec. VIII. While fluid interfaces and gravity have been simulated previously using a lattice Boltzmann model it is important to fully test this model. In particular it is a significant application of gravity to a two phase simulation. Gravity has been applied to a multi-component lattice Boltzmann simulation, see for example Ref. 33, but only in such a way that it acts equally on each fluid. Previously it has been proposed ${ }^{34}$ that gravity can act unequally on both fluids, and shown that this has the effect of producing a different 
density gradient in each fluid. This is the first application where this implementation of gravity allows the simulation of two fluids with different specific gravities. Since the interfacial waves are driven solely by gravity this is a significant new application of this technique. The simulations are also a new application for the binary fluid model which has previously been used to study phenomena such as spinodal decomposition ${ }^{35}$ and the effect of shear on droplets; ${ }^{36}$ simulations which are significantly different from its use here. The lattice Boltzmann approach is derived from the lattice gas technique which has previously been applied to surface gravity waves, ${ }^{37,38}$ however, there are a number of differences which make the lattice Boltzmann model superior. A detailed description of the development of the lattice Boltzmann model from the lattice gas and its advantages over the original scheme are detailed in, for example, Ref. 39. The main advantages applied to our simulations are the removal of noise from the simulations, the Galilean invariance inherent in the simulations and a variable, density independent viscosity. The noise-free results presented here are in contrast to the results obtained from a lattice gas simulation. This not only means that more precise results can be taken from the lattice Boltzmann simulations, but also that the massive amount of averaging required in a lattice gas simulation is no longer required; this considerably reduces the computer resources required. The Galilean invariance of the lattice Boltzmann model used here is also important. In Ref. 38 it is shown that the effect of the lack of Galilean invariance in a lattice gas model can be removed using a scaling technique, as has been done elsewhere, ${ }^{40,41}$ however it is clearly advantageous to use a model where this problem does not arise. It is worth noting that while there are a number of Galilean invariant lattice Boltzmann models for binary fluids, there are none which incorporate a liquid-gas interface which would be required to simulate waves at a free surface. The density-dependent nature of the viscosity in the lattice gas model need not be a problem if the density within each fluid is approximately constant, however it is preferable to use a lattice Boltzmann model in which the viscosity is independent of density and the variable nature of the viscosity increases the range of problems which can be studied. The lattice Boltzmann model has the further advantage that it can be derived from the classical Boltzmann model ${ }^{42}$ which gives it a firm physical base.

In Sec. II the lattice Boltzmann technique is discussed and the model employed here is described. In Sec. III we describe the method used to initialize the waves and results from the simulations are presented in Sec. IV. The theoretical expressions governing linear waves are considered in Sec. V. In Sec. VI we present the results of numerous simulations and compare them with the theory. In Sec. VII we consider the relation between the waves simulated here and waves in physical problems. The work is summarized in Sec. VIII. Throughout this paper the Greek indices $\alpha$ and $\beta$ are used to refer to components and summation over repeated indices will be assumed. Roman indices and the Greek indices $\rho$ and $\Delta$ will be used as labels and no summation will be implicit over repeated indices. When a two-layer fluid is be- ing considered the index " 1 " will refer to the upper fluid and the index " 2 " to the lower fluid.

\section{THE LATTICE BOLTZMANN MODEL}

The lattice Boltzmann model has developed from the lattice gas automaton. ${ }^{43}$ The lattice Boltzmann model employed here uses a hexagonal grid in which each node is connected to its six nearest neighbors along a vector $\boldsymbol{e}_{i}$ $=\sin (\pi i / 3-\pi / 6) \boldsymbol{i}+\cos (\pi i / 3-\pi / 6) \boldsymbol{j}, i=1,2, \ldots, 6$, where $\boldsymbol{i}$ and $\boldsymbol{j}$ are unit vectors in the horizontal and vertical directions respectively. The distribution function $f_{i}$ of particles with velocity $\boldsymbol{e}_{i}$ at node $\boldsymbol{x}$ at time $t$ is evolved according to the BGK Boltzmann equation,

$$
f_{i}\left(\boldsymbol{r}+\boldsymbol{e}_{i}, t+1\right)-f_{i}(\boldsymbol{r}, t)=\frac{-1}{\tau}\left(f_{i}-\bar{f}_{i}\right),
$$

where $\bar{f}_{i}$ are the equilibrium distribution functions and $\tau$ is the relaxation time. The fluid density and momentum are found from the distribution functions as

$$
\rho=\sum_{i=0}^{6} f_{i}
$$

and

$$
\rho u_{\alpha}=\sum_{i=0}^{6} f_{i} e_{i \alpha},
$$

where $f_{0}$ is the rest-particle distribution function and $\boldsymbol{e}_{0}$ is the null vector. The form of the equilibrium distribution function, $\bar{f}_{i}$, is chosen so that it satisfies the conservation of mass and momentum and any other required properties such as Galilean invariance and isotropy. The following equilibrium distribution function ${ }^{44}$ satisfies these criterion,

$\bar{f}_{i}(\boldsymbol{r}, t)$

$$
=\left\{\begin{array}{l}
\rho\left[\frac{1-d_{0}}{6}+\frac{1}{3} \boldsymbol{e}_{i} \cdot \boldsymbol{u}+\frac{2}{3}\left(\boldsymbol{e}_{i} \cdot \boldsymbol{u}\right)^{2}-\frac{u^{2}}{6}\right], \quad i=1, \ldots, 6, \\
\rho\left(d_{0}-u^{2}\right), \quad i=0,
\end{array}\right.
$$

where $d_{0}$ is a constant and $\rho$ and $\boldsymbol{u}$ are given by Eqs. (2) and (3).

\section{A. Boundary conditions in a lattice Boltzmann simulation}

The initial approach to simulating a boundary was to follow the lattice gas method and reflect all distribution functions, at the boundary sites, back along the links they arrived on. Averaging the velocity at the boundary, before and after a collision, gives the required boundary condition $\langle\boldsymbol{u}\rangle=0$. A number of variations on this theme have been proposed, ${ }^{45,46}$ however, such boundary conditions only produce first-order accuracy and have been shown to produce a wall velocity which is a function of the relaxation parameter $\tau^{47}$

Here we apply Dirichlet boundary conditions ${ }^{47}$ for the simple case of a no-slip boundary parallel to $\boldsymbol{e}_{2}$. The evolution of each site requires the values of the distribution func- 
tions, $f_{i}\left(\boldsymbol{r}-\boldsymbol{e}_{i}, t-1\right)$, at the neighboring nodes at the previous time-step to be known. At a boundary site two of these distribution functions $\left(f_{1}\right.$ and $f_{6}$ or $f_{3}$ and $f_{4}$ ) are unknown since they are not defined out with the fluid. These values are found by solving the no-slip boundary condition of zero velocity at the boundary:

$$
f_{1}+2 f_{2}+f_{3}-f_{4}-2 f_{5}-f_{6}=0
$$

and

$$
f_{1}-f_{2}-f_{3}+f_{6}=0 .
$$

Defining the unknown distribution functions in this way means that the no-slip boundary condition is assured at the boundary, at least to second-order accuracy.

\section{B. Binary fluid models}

A number of different approaches have been considered for modeling two similar binary fluids using the lattice Boltzmann technique. ${ }^{48-51}$ Here we consider the model proposed by Orlandini et al. ${ }^{52,53}$ with a body force incorporated. ${ }^{34}$ This binary fluid model is preferred because it is developed from the underlying thermodynamics of phase separation. Gravity has been implemented in other lattice Boltzmann simulations, however, in this study it is solely responsible for driving the simulation. Thus the performance of the model is a significant test for the technique by which gravity is incorporated in the scheme. Rather than considering the densities of the individual fluids, the total density $\rho$ and the density difference or order parameter $\Delta \rho$ are considered. Two distribution functions $f_{i}$ and $\Delta_{i}$ are then used to describe the population of $\rho$ and $\Delta \rho$, respectively, on each of the $i$ links. The evolution of both distribution functions are governed by the single relaxation time Boltzmann equations:

$$
f_{i}\left(\boldsymbol{r}+\boldsymbol{e}_{i}, t+1\right)-f_{i}(\boldsymbol{r}, t)=-\frac{1}{\tau_{\rho}}\left(f_{i}-\overline{f_{i}}\right)+\frac{1}{3} F_{\alpha} e_{i \alpha}
$$

and

$$
\Delta_{i}\left(\boldsymbol{r}+\boldsymbol{e}_{i}, t+1\right)-\Delta_{i}(\boldsymbol{r}, t)=-\frac{1}{\tau_{\Delta}}\left(\Delta_{i}-\overline{\Delta_{i}}\right),
$$

where the body force is given by ${ }^{34}$

$$
\boldsymbol{F}=\left[g_{a}(\rho-\Delta \rho)+g_{b}(\rho+\Delta \rho)\right] \hat{\boldsymbol{e}}_{z},
$$

the density and the order parameter are given by

$$
\rho=\sum_{i} f_{i}, \quad \Delta \rho=\sum_{i} \Delta_{i}
$$

the equilibrium distributions are given by ${ }^{53}$

$$
\begin{aligned}
\bar{f}_{i}= & A+B u_{\alpha} e_{i \alpha}+C u^{2}+D u_{\alpha} u_{\beta} e_{i \alpha} e_{i \beta}+G_{\alpha \beta} e_{i \alpha} e_{i \beta}, \\
& i=1, \ldots, 6 \\
\bar{f}_{0}= & A_{0}+C_{0}, \\
\bar{\Delta}_{i}= & H+K u_{\alpha} e_{i \alpha}+J u^{2}+Q u_{\alpha} u_{\beta} e_{i \alpha} e_{i \beta}, \quad i=1, \ldots, 6,
\end{aligned}
$$

$$
\bar{\Delta}_{0}=H_{0}+J_{0} .
$$

Here $\hat{\boldsymbol{e}}_{z}$ is a unit vector in the vertical direction, $g_{a}$ and $g_{b}$ determine the strength of the gravitational interaction and the coefficients in the expansions of the equilibrium distributions $\operatorname{are}^{53}$

$$
\begin{aligned}
& A_{0}=\rho-6 A, \quad A=\left(\rho T-\kappa \Delta \rho \nabla^{2} \Delta \rho / 2-\kappa \rho \nabla^{2} \rho / 2\right) / 3, \\
& B=\rho / 3, \quad C=-\rho / 6, \quad C_{0}=-\rho, \quad D=2 \rho / 3, \\
& G_{x y}=G_{y x} \\
& =\frac{2 \kappa}{3}\left[\frac{\partial \rho}{\partial x} \frac{\partial \rho}{\partial y}+\frac{\partial \Delta \rho}{\partial x} \frac{\partial \Delta \rho}{\partial y}\right], \quad H_{0}=\Delta \rho-6 H, \\
& G_{x x}=-G_{y y}=\frac{\kappa}{3}\left[\left(\frac{\partial \rho}{\partial x}\right)^{2}-\left(\frac{\partial \rho}{\partial y}\right)^{2}\right] \\
& +\left[\left(\frac{\partial \Delta \rho}{\partial x}\right)^{2}-\left(\frac{\partial \Delta \rho}{\partial y}\right)^{2}\right] \\
& H=\Gamma \Delta \mu / 3, \quad K=\Delta \rho / 3, \quad J=-\Delta \rho / 6, \\
& J_{0}=-\Delta \rho, \quad Q=2 \Delta \rho / 3 \text {. }
\end{aligned}
$$

The chemical potential difference is given by

$$
\Delta \mu=-\frac{\Lambda}{2} \frac{\Delta \rho}{\rho}+\frac{T}{2} \log \left(\frac{1+\Delta \rho / \rho}{1-\Delta \rho / \rho}\right)-\kappa \nabla^{2} \Delta \rho .
$$

The interfacial energy $\kappa$, the temperature $T$, the interaction strength parameter $\Lambda$, the mobility $\Gamma$ and the relaxation times $\tau_{\rho}$ and $\tau_{\Delta}$ are free to be varied to change the properties of the binary fluid. Here these parameters have the values $\kappa=0.001, T=0.5, \Lambda=1.1, \Gamma=0.1$ and $\tau_{\Delta}=0.789$. The relaxation parameter for the whole fluid was varied in the simulations to change the fluid viscosity which is given by

$$
\nu=\frac{2 \tau_{\rho}-1}{8} .
$$

It can be shown ${ }^{53}$ that such a fluid satisfies the continuity equation for the whole fluid,

$$
\partial_{t} \rho+\partial_{\alpha} \rho u_{\alpha}=0
$$

the Navier-Stokes equation for the whole fluid,

$$
\partial_{t} \rho u_{\alpha}+\partial_{\beta} \rho u_{\alpha} u_{\beta}=-\partial_{\alpha} p_{0}+\nu \partial_{\beta} \partial_{\beta} \rho u_{\alpha}+\partial_{\alpha} \zeta \partial_{\beta} \rho u_{\beta}
$$

and the convection-diffusion equation for the density difference,

$$
\partial_{t} \Delta \rho+\partial_{\alpha} \Delta \rho u_{\alpha}=\Gamma \theta \partial_{\beta} \partial_{\beta} \Delta \mu-\theta \partial_{\alpha}\left(\frac{\Delta \rho}{\rho} \partial_{\beta} P_{\alpha \beta}\right),
$$

where

$$
\nu=\frac{2 \tau_{\rho}-1}{8}, \quad \zeta=\left(\tau_{\rho}-\frac{1}{2}\right)\left(\frac{1}{2}-\frac{\partial p_{0}}{\partial \rho}\right)
$$

and

$$
\theta=\left(\tau_{\Delta}-\frac{1}{2}\right)
$$




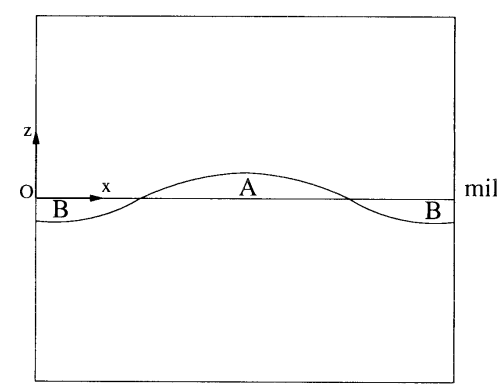

FIG. 1. The initialization of a standing wave.

When the values of $\Lambda$ and $T$ are restricted to $\Lambda=1.1$ and $T=0.5$ the ratio $|\Delta \rho / \rho| \simeq 0.5$ at all depths ${ }^{34}$ so the value of $g$ in both fluids is

$$
g_{1}=\frac{3}{2} g_{a}+\frac{1}{2} g_{b}, \quad g_{2}=\frac{1}{2} g_{a}+\frac{3}{2} g_{b} .
$$

With $g_{a}<g_{b}$ we find $g_{1}<g_{2}$ and the fluid separates into two fluids with a horizontal interface between them. Gravity acts with strength $g_{1}$ in the upper fluid where $\Delta \rho<0$ and with strength $g_{2}$ in the lower fluid where $\Delta \rho>0$.

\section{Lattice Boltzmann model for interfacial waves}

The interfacial wave simulations presented here were performed using the immiscible, binary fluid model described by Eqs. (7)-(16) with a gravitational interaction introduced through Eq. (9). Dirichlet boundary conditions were applied at all solid boundaries by solving Eqs. (5) and (6) for the required distribution functions. All the simulations were performed with $\Lambda=1.1, T=0.5, \Gamma=0.1, \tau_{\Delta}=0.789$ and $\kappa$ $=0.001$. These parameters produce immiscible fluids separated by a sharp interface.

\section{STANDING WAVE INITIALIZATION}

The ability of the model to simulate interfacial waves was investigated using standing waves. These were initialized on grids with the same number of grid points in the horizontal and vertical directions, the grid being orientated with $\boldsymbol{e}_{2}$ and $\boldsymbol{e}_{5}$ horizontal. The grid was initialized with $\rho$ $=1.0$ and divided into two by a horizontal line near the center. The line was taken either to be at the center of the grid or, when a large gravitational force was being applied, near the center with the bottom section slightly larger than the top. The order parameter $\Delta \rho$ was set to -0.5 above the line and to +0.5 below the line. A solid no-slip boundary was set at the bottom and top of the fluid and continuous boundary conditions applied at the other edges. The fluid was then allowed to evolve with $\tau_{\rho}=200.0$ for 8,000 time-steps. This allows the fluids to reach an equilibrium state where the density gradient, produced by the body force, in each fluid is established. The height of the interface between the two fluids was then found; this is the mean interface level (mil). A sinusoidal interface was then imposed between the fluids about the previous interface, as shown in Fig. 1, and the sign of $\Delta \rho$ was switched in regions $A$ and $B$. The coordinate system is also shown in Fig. 1. The sinusoidal interface has a wavelength equal to the length of the grid and an amplitude of $20 \sqrt{3} / 2$. Using this initialization method the density gradients are set correctly and any initial $z$-velocity, produced by the creation of these gradients, has been damped, by the high viscosity, to a negligible magnitude. The interfacial energy used in all simulations is $\kappa=0.001$ which gives a sharp interface between the two fluids. When an $l$ by $l$ site grid is used and the mean interface level is exactly in the middle of the grid, then the wavelength is $\lambda=l$ and the depth of the two fluids are $h_{1}=h_{2}=\sqrt{3} l / 4$ (since the vertical separation of sites on the hexagonal grid used here is $\sqrt{3} / 2$ ). Thus $\tanh \left(k h_{1}\right)=\tanh \left(k h_{2}\right)=0.99$ and both fluids can be considered as being deep.

\section{STANDING WAVE SIMULATIONS}

Here we present the results of some simulations and consider the accuracy of the values obtained. The simulations were run on the Connection Machine CM-200 at the University of Edinburgh.

\section{A. Velocity and density measurements}

A velocity vector map and the position of the interface, seen through the value of the order parameter, is shown at selected times in Figs. 2-4 for a typical interfacial wave on a 256 by 256 grid with $g_{1}=2.5 \times 10^{-4}, g_{2}=3.5 \times 10^{-4}, \lambda$ $=256$ and $\nu=0.05$. The interface is seen to be sharp as is expected when $\kappa=0.001$. The velocity is measured in latticesteps per time-step. The whole of the 256 by 256 grid is shown in each vector map with the same scale in the $x$ - and $z$-directions. The scale of the $x$ - and $z$-directions is also the same for the plot of the order parameter but only half of the grid is shown. The results in Figs. 2-4 show qualitatively the expected wave motion.

In a standard lattice Boltzmann model any numerical error in the velocity and density measurements come from floating point errors in the calculation of the distribution function $f_{i}$ and should be negligible. It is important that the fluid velocities in the simulation remain small since the lattice Boltzmann model will only mimic a real fluid in this regime because terms $O\left(u^{3}\right)$ are neglected in the derivation of the Navier-Stokes equation. Here the largest velocities are $O(0.01)$ so the model should give an accurate realization of the fluids being simulated. The main source of error is through the introduction of the differentials of the density and order parameters in Eqs. (15) and (16). These are calculated by finite-difference approximations. ${ }^{53}$ The order parameter changes rapidly in the region of the interface, particularly when the interface is relatively sharp, so the largest source of error will be in the approximation of its derivatives at the interface. Small spurious velocities have been observed in the interface region ${ }^{53}$ when the interface has width $\sim 5$ lattice-steps $(\kappa=0.2)$. The magnitude of the velocities was $1.09 \times 10^{-4}$ for the value of $\tau_{\Delta}$ used here. In the simulations presented here, where $\kappa=0.001$, small spurious velocities were also observed at the interface but only when the fluid velocity close to the interface was small, $\simeq 0.0007$ lattice-steps per time-step. Small spurious velocities at the interface are a feature of both lattice Boltzmann and finitedifference simulations of interfaces. 

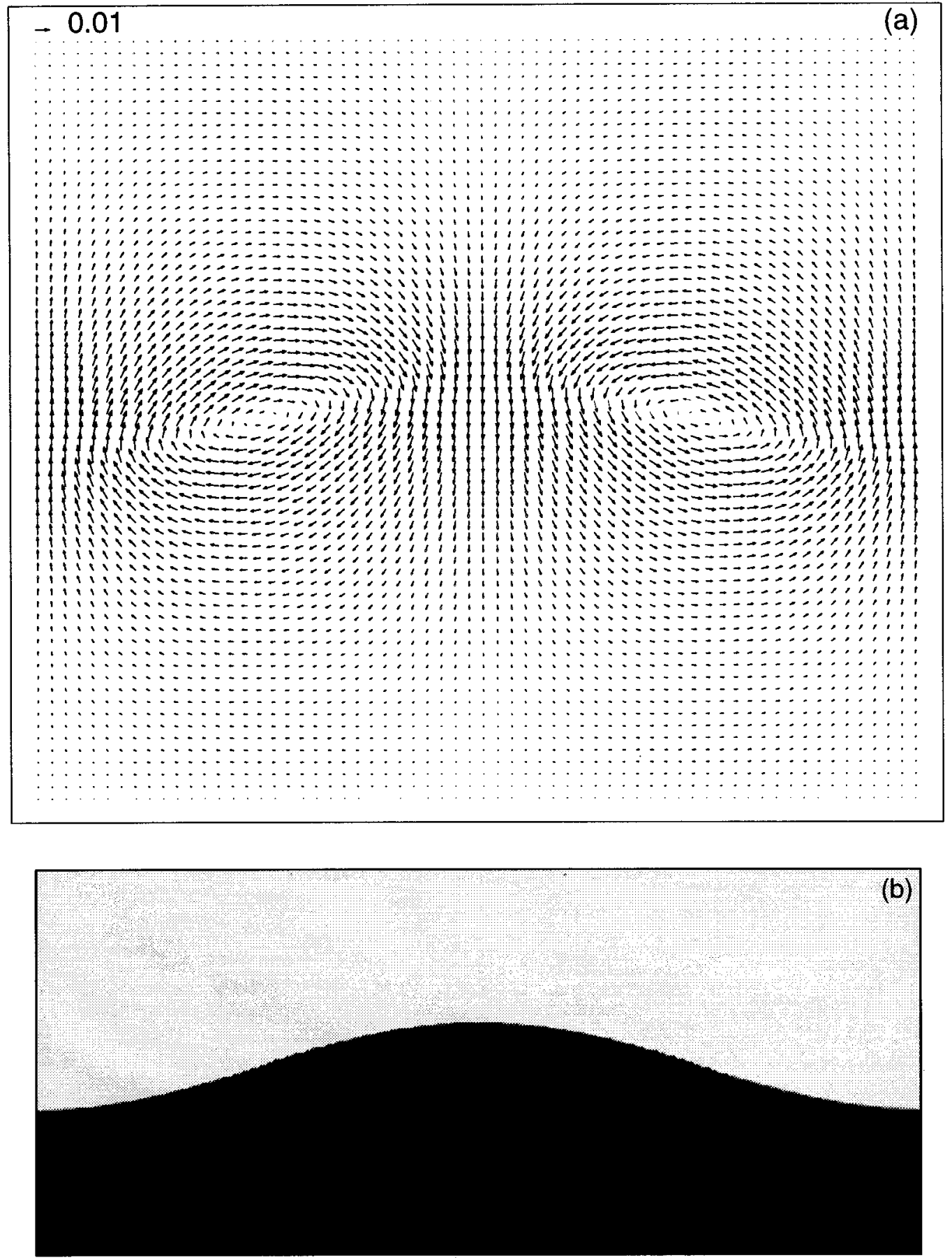

FIG. 2. (a) Velocity vector plot and (b) the order parameter at $t \simeq T / 8$ for an interfacial standing wave on a 256 by 256 grid with $g_{1}=2.5 \times 10^{-4}, g_{2}=3.5$ $\times 10^{-4}, \lambda=256$ and $\nu=0.05$. Only half the grid, centered on the interface, is shown for the order parameter.

\section{B. Frequency and damping rate measurements}

During the simulations the height of the interface, above the bottom boundary, was found every forty time-steps at the center of the wave, $x=\lambda / 2$. The height of the wave was taken to be the height of the highest site containing the denser fluid. This gives a time series record of the wave height at the center which was then fitted to a curve of the form

$$
A e^{-\alpha t} \cos (\omega t+\psi)+c .
$$

This was done using a modified Gauss-Newton $\operatorname{algorithm~}^{54,55}$ where the parameters $A, \alpha, \omega, \psi$ and $c$ are the parameters found by the fitting process. The sum of the square of the deviation of the data from the fitted curve, $e$, was also computed and this gives a measure of the accuracy of the curve fitting process and the fitted parameters. The two parameters in which we are mainly interested are the frequency $\omega$ and the damping rate $\alpha$. The fitted parameter $c$ should correspond to the mean interface level $h_{2}$ which was 

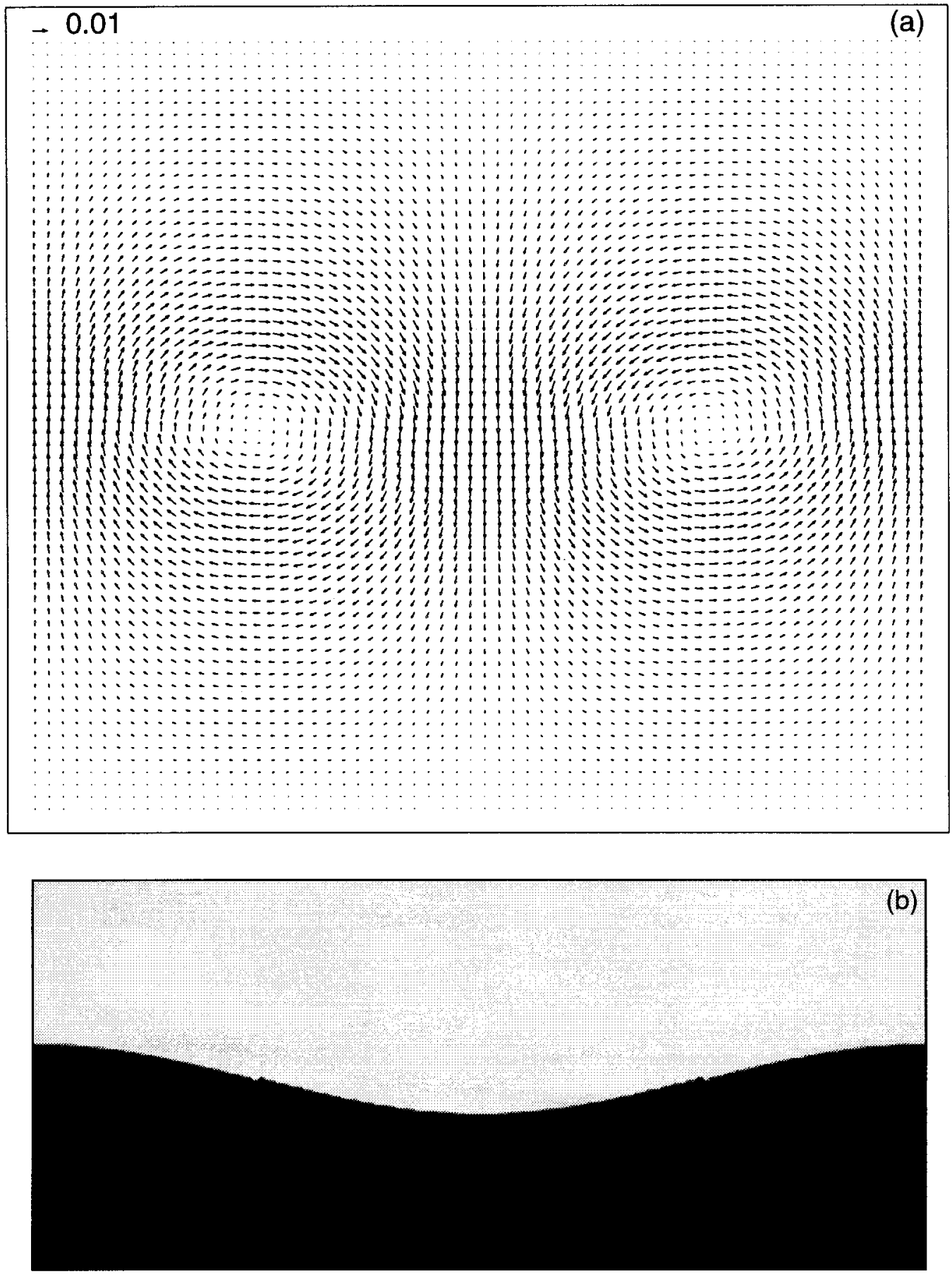

FIG. 3. (a) Velocity vector plot and (b) the order parameter at $t \simeq 3 T / 8$ for an interfacial standing wave on a 256 by 256 grid with $g_{1}=2.5 \times 10^{-4}, g_{2}$ $=3.5 \times 10^{-4}, \lambda=256$ and $\nu=0.05$. Only half the grid, centered on the interface, is shown for the order parameter.

used in initializing the wave. In practice it is shifted slightly, even when $\kappa$ is small and the interface is sharp. The curve fitting process was applied using an equation of the form

$$
A e^{-\alpha t} \cos (\omega t+\psi)+h_{2},
$$

where $h_{2}$ is fixed and the other parameters were found as before. The results obtained for $\omega$ and $\alpha$ were found to be within $1 \%$ of their previous values. The value of $e$ was increased by $\sim N\left|h_{2}-c\right|^{2}$, where $N$ is the number of points.
This means that $e$ is no longer a good measure of the fit of the other parameters. Thus it was decided to include $c$ in the set of fitted parameters. Similarly the value of $A$ should be related to the initial deformation amplitude. It is, however, slightly different and can influence the accuracy with which the other parameters are found. If $A$ is fixed, in Eq. (24), to an incorrect value there is little change in $\omega$ but there can be a significant change in $\alpha$. If $A$ is set too large the value found for $\alpha$ can be seen to be too large, the values of the fitted 

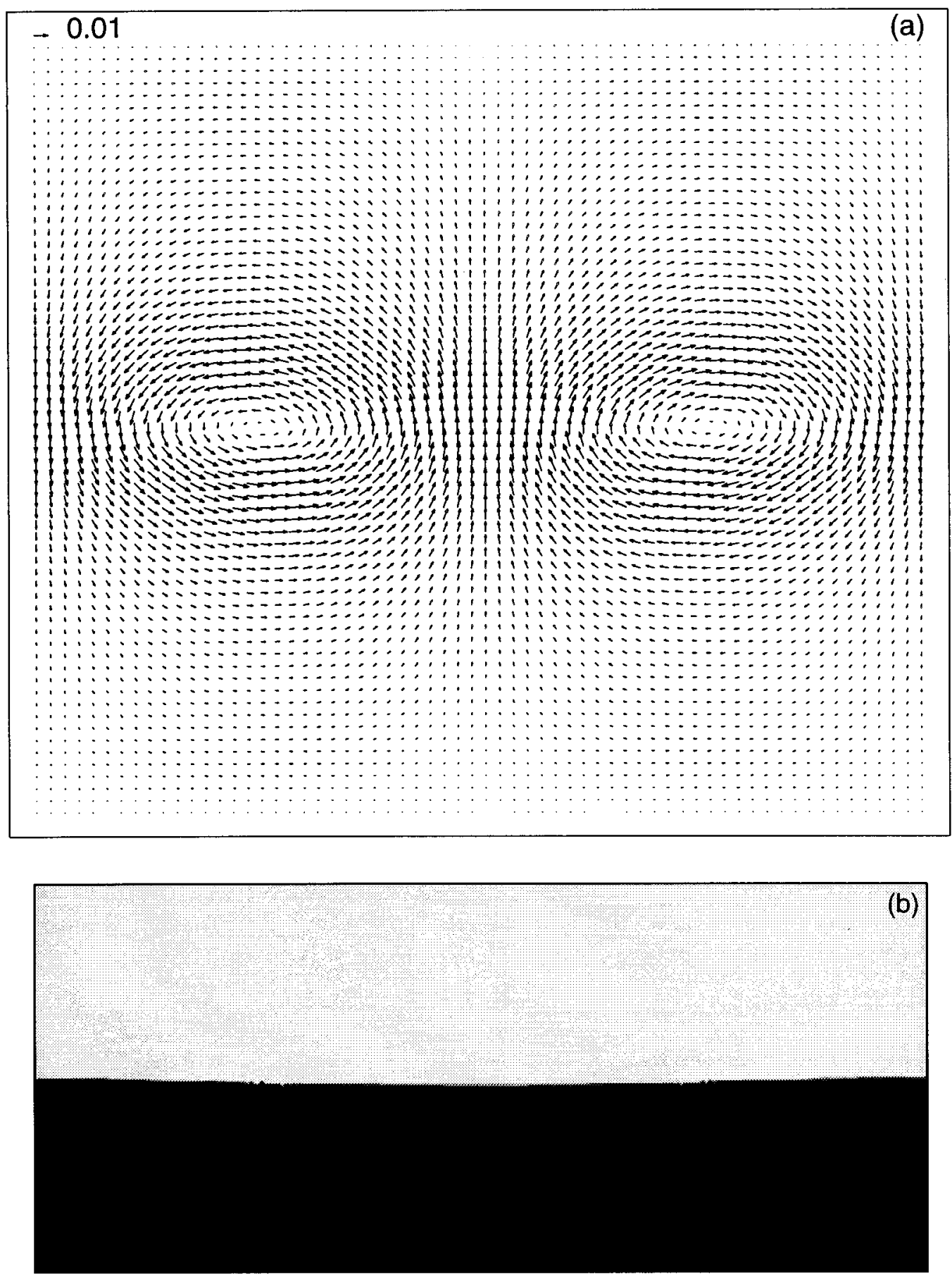

FIG. 4. (a) Velocity vector plot and (b) the order parameter at $t \simeq 6 T / 8$ for an interfacial standing wave on a 256 by 256 grid with $g_{1}=2.5 \times 10^{-4}, g_{2}$ $=3.5 \times 10^{-4}, \lambda=256$ and $\nu=0.05$. Only half the grid, centered on the interface, is shown for the order parameter.

curve being greater than the data points for small times and smaller than the data points for large times. If $A$ is set too small the value of $\alpha$ is correspondingly too small. Thus it is important that $A$ has the correct value and this is best achieved by allowing it to be found by the fitting routine. A phase difference $\psi$ is also included in Eq. (24). This accounts for any discrepancy in the initialization procedure. The main error in the initialization is the profile of the density and the order parameter at the interface. The values found for $\psi$ were no larger than $1 \%$ of $2 \pi$ suggesting that the initialization method is adequate. The average value found for $e$ from over a hundred different waves, each with either 1,000 or 900 data points, was found to be 85 . This corresponds to a root mean squared difference between the data and the fitted curve of 0.31 lattice-steps.

To assess the size of this error the values of the curve in Eq. (24) were calculated, using the typical parameters: $A$ $=20 \sqrt{3} / 2, \alpha=5 \times 10^{-5}, \omega=2 \pi / 6,000, \psi=0$ and $c=128$, at times $t_{i}=40 i, i=1,2, \ldots, 1,000$. The sum of the square of the difference between these 1,000 values and their nearest inte- 


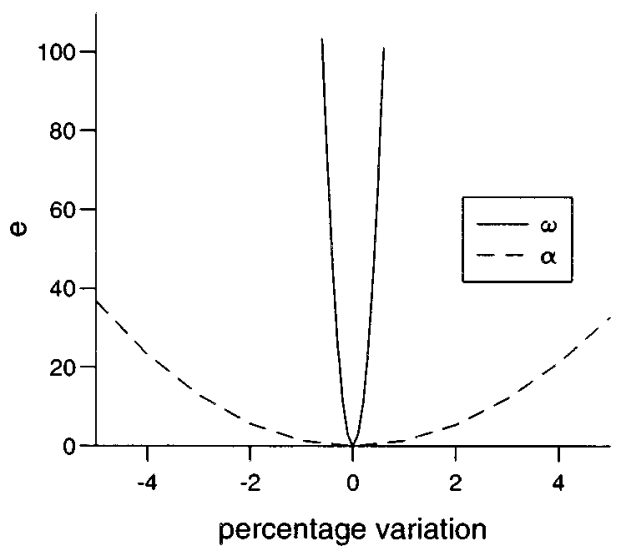

FIG. 5. The sum of the square of the deviation between two curves of the form of Eq. (24) when the value of either $\omega$ or $\alpha$ has been varied, in one of the curves, by a small percentage from $2 \pi / 6000$ and $5 \times 10^{-5}$, respectively. The sum is over 1000 different values taken at times $t=40 i, i$ $=1,2, \ldots, 1000$. The values of the other parameters used are $A$ $=20 \sqrt{3} / 2, \quad \psi=0$ and $c=128$.

ger value was then calculated and found to be 60.2. This is the value of $e$ produced solely by the spatially discrete nature of the model. The values of $e$ obtained from the curve fitting process are not significantly larger suggesting that Eq. (24) correctly describes the interface and that the fitted parameters are reasonably accurate.

To investigate how a small error in $\omega$ or $\alpha$ will affect the error parameter $e$, the values of the curve at each of the 1,000 times calculated above was compared to the value calculated when either $\omega$ or $\alpha$ was varied by a small amount. The sum of the square of these differences, $e$, was calculated and is shown plotted against the percentage variation in either $\omega$ or $\alpha$ in Fig. 5. Thus the difference between the average value of $e$ found from curve fitting, 85, and the expected value of 60 , due to the discrete nature of the grid, corresponds to an error in $\omega$ of about $0.3 \%$ or an error in $\alpha$ of about $4 \%$.

A typical set of results is shown in Fig. 6 where only every tenth result has been plotted. The wave was simulated using $g_{1}=1.25 \times 10^{-4}, g_{2}=1.75 \times 10^{-4}, \nu=0.05$ and $\lambda$ $=256$. The best fit curve through all the data points is also shown. The close agreement between the data points and the fitted curve can be seen clearly. A number of longer simulations were also run. These simulations were generally performed on a larger grid because of the high rate of damping observed when a wavelength of 256 is used. A typical example is shown in Fig. 7 which was simulated on a 512 by 512 grid. Only points every 400 time-steps have been marked. The wave was simulated with $g_{1}=2.5 \times 10^{-4}, g_{2}$ $=3.5 \times 10^{-4}, \nu=0.05$ and $\lambda=512$. The wave was allowed to evolve for $1 \times 10^{5}$ time-steps and was observed to oscillate for almost twelve periods in this time. The agreement between the results and the best fit curve through the points is good although the discrepancy becomes larger towards the end of the simulation when the amplitude of the waves is only a few lattice-steps. The error is slightly larger than that found earlier when a shorter time period was considered. Here $e=2531$ giving a root mean square deviation of 1.0

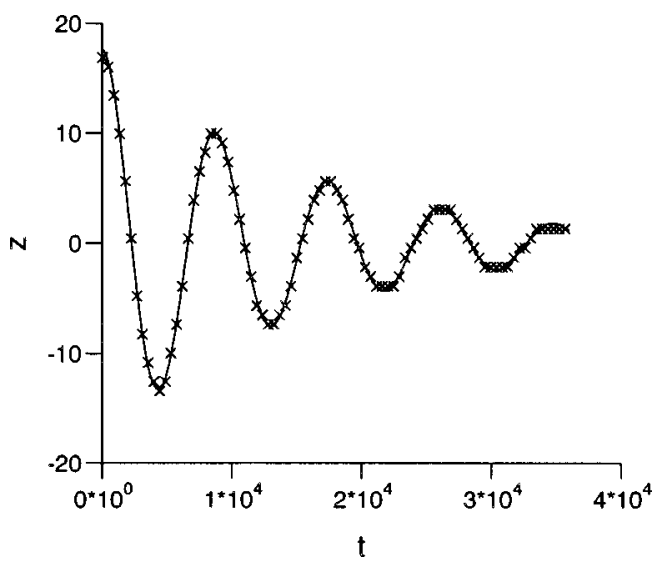

FIG. 6. The wave's height, at $x=\lambda / 2$, as a function of time for a standing wave with $\lambda=256, \nu=0.05, g_{1}=1.25 \times 10^{-4}$ and $g_{2}=1.75 \times 10^{-4}$. Only points every 400 time-steps have been marked. Also shown is the best fit curve through all the data points. This has the forms of Eq. (24) with $A$ $=17.7, \alpha=6.52 \times 10^{-5}, \omega=7.49 \times 10^{-4}, \psi=0.005$ and $c=110$.

lattice spacing. The results in Figs. 6 and 7 both show the interface between the fluids oscillating in a sinusoidal manner with a regular period and a decaying amplitude in the manner we would expect when there are standing waves produced at the interface.

\section{THEORETICAL RESULTS FOR INTERFACIAL WAVES}

We consider two fluids of depth $h_{1}$ and $h_{2}$ with densities $\rho_{1}$ and $\rho_{2}, \rho_{1}<\rho_{2}$, separated by a sharp interface. Let the origin be at the interface with the $x$-coordinate horizontal and the $z$-coordinate vertically upwards. In this section we briefly review the linear wave equations governing interfacial gravity waves. Before considering these equations we must first consider the potential density.

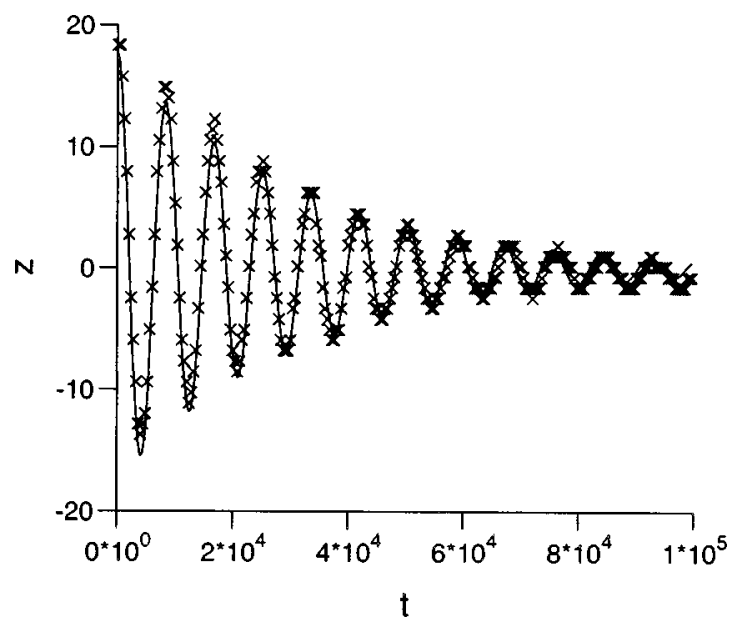

FIG. 7. The wave's height, at its center, as $x=\lambda / 2$ of time for a standing wave with $\lambda=512, \nu=0.05, g_{1}=2.5 \times 10^{-4}$ and $g_{2}=3.5 \times 10^{-4}$. Only points every 400 time-steps have been marked. Also shown is the best fit curve through all the data points. This has the forms of Eq. (24) with $A$ $=17.6, \alpha=3.22 \times 10^{-5}, \omega=7.23 \times 10^{-4}, \psi=-0.07$ and $c=237$. 


\section{A. The potential density}

The density of a fluid is a function of the pressure $p$, temperature $T$ and, for a liquid, the salinity $\chi$, defined as the proportion by mass of the dissolved salts. ${ }^{56}$ Consider a particle at height $z$, where the density is $\rho(z)$, in a fluid with no temperature or salinity variation. The pressure at height $z$ is given by the hydrostatic pressure equation ${ }^{56}$

$$
\frac{d p(z)}{d z}=-\rho(z) g .
$$

If the particle moves slightly to height $z+\xi$ then the pressure acting on the particle and the fluid density will change to $p(z+\xi)$ and $\rho(z+\xi)$, respectively, and the particle will remain in equilibrium. When we are considering internal waves we are concerned, not with the actual density, but with the excess or potential density defined ${ }^{57,58}$ as the density the fluid would have if compressed adiabatically, with constant salinity, to a reference pressure $p_{0}$. This can be expressed, ${ }^{58}$

$$
\rho_{\mathrm{pot}}=\rho-\int_{p_{0}}^{p}\left(\frac{\partial \rho}{\partial p}\right)_{\chi S} d p,
$$

where $S$ is the entropy. This means that a fluid which has a density gradient produced solely by gravity has $\rho_{\text {pot }}$ constant and no internal wave motion will occur. In practice internal waves only occur between fluids with different densities or when there is a change in temperature or a change in salinity with depth.

\section{B. Interfacial waves between two inviscid fluids}

Gravity wave motion between immiscible, homogeneous, incompressible fluids can, provided any disturbances are small, be described by linear wave theory in a manner analogous to linear surface waves. If the fluids are irrotational then the irrotational velocities $\boldsymbol{u}_{0}=\left(u_{0}, w_{0}\right)$ can be found by solving the Laplace equation, $\nabla^{2} \phi_{i}=0$ where $\phi_{i}$ is the velocity potential in fluid $i$, subject to the standard boundary conditions.

This gives the velocity as

$u_{0}=-\frac{\omega_{0} a \cosh \left[k\left(z-h_{1}\right)\right]}{\sinh \left(k h_{1}\right)} \sin (k x) \sin \left(\omega_{0} t\right), \quad 0<z<h_{1}$,

$u_{0}=\frac{\omega_{0} a \cosh \left[k\left(z+h_{2}\right)\right]}{\sinh \left(k h_{2}\right)} \sin (k x) \sin \left(\omega_{0} t\right), \quad 0>z>-h_{2}$,

$w_{0}=\frac{\omega_{0} a \sinh \left[k\left(z-h_{1}\right)\right]}{\sinh \left(k h_{1}\right)} \cos (k x) \sin \left(\omega_{0} t\right)$,

$0<z<h_{1}$

and

$$
\begin{aligned}
w_{0}= & -\frac{\omega_{0} a \sinh \left[k\left(z+h_{2}\right)\right]}{\sinh \left(k h_{2}\right)} \cos (k x) \sin \left(\omega_{0} t\right), \\
& 0>z>-h_{2} .
\end{aligned}
$$

The frequency and wavenumber satisfy the dispersion relation

$$
\frac{\omega_{0}^{2} \rho_{1}}{k \tanh \left(k h_{1}\right)}+\frac{\omega_{0}^{2} \rho_{2}}{k \tanh \left(k h_{2}\right)}-\left(\rho_{2}-\rho_{1}\right) g=0 .
$$

If the interface is far from the bed and the free surface so that $\tanh \left(k h_{1}\right) \simeq 1$ and $\tanh \left(k h_{2}\right) \simeq 1$ this can be written in a form analogous to the dispersion relation for surface waves,

$$
\omega_{0}^{2}=g^{\prime} k,
$$

where $g^{\prime}$ is the reduced gravity and is defined by

$$
g^{\prime} \equiv \frac{\left(\rho_{2}-\rho_{1}\right)}{\left(\rho_{2}+\rho_{1}\right)} g .
$$

Provided both fluids are deep there will be no attenuation of the wave amplitude or the wave velocities.

\section{Interfacial waves between viscous fluids}

If the fluids are in fact viscous this will affect the waves velocities, the damping rate and the wave frequency. The linearized Navier-Stokes equation permits the velocity to be split into a potential part, $\boldsymbol{u}_{0}=\boldsymbol{\nabla} \phi$, and a rotational part, $\boldsymbol{U}$ $=(U, W)$. The solution for $\boldsymbol{u}_{0}$ is simply the inviscid solution which has already been given in Eqs. (28)-(31). Provided

$$
\epsilon_{i}=\frac{\omega^{2}}{g}\left(\frac{\nu_{i}}{\omega}\right)^{1 / 2}
$$

is small in both fluids; the motion is essentially irrotational except near viscous boundary layers which have a thickness of order $\left(\nu_{i} / \omega\right)^{1 / 2}$ and which occur at solid boundaries and at the interface. Thus, following Refs. 59, 60, we look for solutions for $\boldsymbol{U}$ which satisfy the Navier-Stokes equation for the rotational part of the velocity,

$$
\frac{\partial \boldsymbol{U}_{i}}{\partial t}=\nu_{i} \nabla^{2} \boldsymbol{U}_{i}
$$

and the appropriate boundary conditions. We only consider solutions which have a significant value in the boundary layer and a negligible value outside the boundary layer. Since we expect ${ }^{61,60}$ that $W_{i}$ will be an order of magnitude smaller then $U_{i}$ we will solve for $U_{i}$, the component in the direction of wave propagation. The solution for $W_{i}$ can then be found by integrating $U_{i} \cdot{ }^{60}$ In the area of the interface we find solutions ${ }^{62,60}$

$$
U_{1}=C_{1} \exp \left[-(1+i)\left(\frac{\omega}{2 \nu_{1}}\right)^{1 / 2} z\right] e^{i k x} \sin (\omega t)
$$

and

$$
U_{2}=C_{2} \exp \left[(1+i)\left(\frac{\omega}{2 \nu_{2}}\right)^{1 / 2} z\right] e^{i k x} \sin (\omega t),
$$

while at the solid boundaries we find solutions,

$$
U_{1}^{\prime}=C_{1}^{\prime} \exp \left[(1+i)\left(\frac{\omega}{2 \nu_{1}}\right)^{1 / 2}\left(z-h_{1}\right)\right] e^{i k x} \sin (\omega t)
$$

and 


$$
U_{2}^{\prime}=C_{2}^{\prime} \exp \left[-(1+i)\left(\frac{\omega}{2 \nu_{2}}\right)^{1 / 2}\left(z+h_{2}\right)\right] e^{i k x} \sin (\omega t) .
$$

The amplitude terms are given by

$$
\begin{aligned}
& C_{1}=\frac{-i f^{\prime}}{1+f^{\prime}}\left[\frac{\omega a}{\sinh \left(k h_{1}\right)} \cosh \left(k h_{1}\right)+\frac{\omega a}{\sinh \left(k h_{2}\right)} \cosh \left(k h_{2}\right)\right], \\
& C_{2}=\frac{i}{1+f^{\prime}}\left[\frac{\omega a}{\sinh \left(k h_{1}\right)} \cosh \left(k h_{1}\right)+\frac{\omega a}{\sinh \left(k h_{2}\right)} \cosh \left(k h_{2}\right)\right], \\
& C_{1}^{\prime}=\frac{-i \omega a}{\sinh \left(k h_{1}\right)} \text { and } C_{2}^{\prime}=\frac{i \omega a}{\sinh \left(k h_{2}\right)},
\end{aligned}
$$

where $f^{\prime}=\left(\rho_{2} / \rho_{1}\right)\left(\nu_{2} / \nu_{1}\right)^{1 / 2}$. The final solution for $\boldsymbol{u}$ can be found by summing all the horizontal velocity terms. In doing this we include the damping factor $\exp (-\alpha t)$ by expressing the amplitude as $A \exp (-\alpha t)$. This gives

$$
\begin{aligned}
u_{1}= & \frac{A \omega}{\sinh (k h)} \exp (-\alpha t)\{-\cosh [k(z-h)] \sin (k x) \sin (\omega t) \\
& +\frac{2 f}{(1+f)} \cosh (k h) \sin \left[k x-\left(\frac{\omega}{2 \nu}\right)^{1 / 2} z\right] \sin (\omega t) \\
& \times \exp \left[-\left(\frac{\omega}{2 \nu}\right)^{1 / 2} z\right]+\sin \left[k x+\left(\frac{\omega}{2 \nu}\right)^{1 / 2}(z-h)\right] \\
& \left.\times \sin (\omega t) \exp \left[\left(\frac{\omega}{2 \nu}\right)^{1 / 2}(z-h)\right]\right\}
\end{aligned}
$$

and

$$
\begin{aligned}
u_{2}= & \frac{A \omega}{\sinh (k h)} \exp (-\alpha t)\{\cosh [k(z+h)] \sin (k x) \sin (\omega t) \\
& -\frac{2}{(1+f)} \cosh (k h) \sin \left[k x+\left(\frac{\omega}{2 \nu}\right)^{1 / 2} z\right] \sin (\omega t) \\
& \times \exp \left[\left(\frac{\omega}{2 \nu}\right)^{1 / 2} z\right]-\sin \left[k x-\left(\frac{\omega}{2 \nu}\right)^{1 / 2}(z+h)\right] \\
& \left.\times \sin (\omega t) \exp \left[-\left(\frac{\omega}{2 \nu}\right)^{1 / 2}(z+h)\right]\right\},
\end{aligned}
$$

where we have set $h_{1}=h_{2}=h$ and $\nu_{1}=\nu_{2}=\nu$. The vertical velocity can be found from the continuity equation and is

$$
w_{1}=-\int_{-h}^{0} \frac{\partial u_{1}}{\partial x} d z-\int_{0}^{z} \frac{\partial u_{1}}{\partial x} d z \text { and } w_{2}=-\int_{-h}^{z} \frac{\partial u_{1}}{\partial x} d z .
$$

It can easily be seen that integrating the differential of the first term in Eqs. (42) and (38) gives the inviscid solution $w_{0}$. The integral of the differential of the second and third terms are smaller than the original terms by a factor $k \sqrt{(2 \nu / \omega)}$ and so give only a very small correction term.

The affect of the viscosity on the wave amplitude and the frequency was investigated by Harrison ${ }^{15}$ who solves the linearized Navier-Stokes equation to obtain the following 'frequency' equation for the complex frequency $\sigma$ :

$$
\begin{aligned}
\sigma= & \pm\left\{\left[\frac{g k\left(\rho_{2}-\rho_{1}\right)}{\left(\rho_{2}+\rho_{1}\right)}\right]^{1 / 2}\right. \\
& \left.-\left[\frac{g k\left(\rho_{2}-\rho_{1}\right)}{\left(\rho_{2}+\rho_{1}\right)}\right]^{1 / 4} \frac{\sqrt{2} k \rho_{2} \rho_{1} \sqrt{\nu_{2} \nu_{1}}}{\left(\rho_{2}+\rho_{1}\right)\left(\rho_{2} \sqrt{\nu_{2}}+\rho_{1} \sqrt{\nu_{1}}\right)}\right\} \\
& +i\left\{\frac{g k\left(\rho_{2}-\rho_{1}\right)}{\left(\rho_{2}+\rho_{1}\right)}\right]^{1 / 4} \frac{\sqrt{2} k \rho_{2} \rho_{1} \sqrt{\nu_{2} \nu_{1}}}{\left(\rho_{2}+\rho_{1}\right)\left(\rho_{2} \sqrt{\nu_{2}}+\rho_{1} \sqrt{\nu_{1}}\right)} \\
& \left.+2 k^{2} \frac{\nu_{2}^{2} \rho_{2}^{3}+\nu_{1}^{2} \rho_{1}^{3}}{\left(\rho_{2}+\rho_{1}\right)\left(\rho_{2} \sqrt{\nu_{2}}+\rho_{1} \sqrt{\nu_{1}}\right)^{2}}\right\} .
\end{aligned}
$$

Expressing $\sigma$ in the form $\sigma= \pm\left(\omega_{0}-\omega^{\prime}\right)-\mathrm{i} \alpha$, where $\omega_{0}$ is the frequency of an inviscid wave, and considering two fluids with the same viscosity, $\nu_{1}=\nu_{2}=\nu$, and with densities given by $\rho_{1}=\rho$ and $\rho_{2}=f \rho$, a series expansion, in terms of $\sqrt{\nu}$, can be made for $\sigma$. To order $O(\nu)$ the solution is ${ }^{15}$

$$
\omega^{\prime}=\left(\omega_{0}\right)^{1 / 2} \frac{\sqrt{2} k f \sqrt{\nu}}{(1+f)^{2}}
$$

and

$$
\alpha=\left(\omega_{0}\right)^{1 / 2} \frac{\sqrt{2} k f \sqrt{\nu}}{(1+f)^{2}}+\frac{2 k^{2}\left(1+f^{3}\right) \nu}{(1+f)^{3}} .
$$

This means that the wave frequency, $\omega=\omega_{0}-\omega^{\prime}$ (and hence also the celerity $c=\omega / k$ ) is reduced by an amount $\omega^{\prime}$ from the inviscid solution and the wave amplitude $a$ is replaced by a decaying exponential: $A \exp (-\alpha t)$. In many situations $\omega^{\prime}$ and $\alpha$ are small and of a similar size. In such situations the frequency is only altered slightly from its inviscid value however the damping term $\exp (-\alpha t)$ can produce a large effect when accumulated over large times. As noted by Harrison ${ }^{15}$ his solution is a series expansion in $\sqrt{\nu}$, correct to order $O(\nu)$ and also an expansion in $k$, correct to order $O\left(k^{2}\right)$. This solution is in agreement with the solution of Johns ${ }^{16}$ which was found by considering separate solutions inside and outside the boundary layer, in a manner akin to the treatment of the velocities at the start of this section, and matching the two solutions. The solution of Johns ${ }^{16}$ is only to first-order in $\sqrt{\nu}$.

\section{RESULTS AND COMPARISON WITH THEORY}

In this section we present the results of numerous simulations and compare the results with the theoretical expressions. The simulations were all performed on the Connection Machine CM-200 at the University of Edinburgh. In each simulation the wave amplitude was initialized to $A$ $=20 \sqrt{3} / 2$.

\section{A. The density gradient, the potential density, the relative density and the gravitational strength}

In Eq. (10) the fluid density was defined as $\rho=\Sigma_{i} f_{i}$. In Eq. (7) gravity was introduced into the model. When this is done the density becomes a function of depth, ${ }^{34}$ and the gradient $\partial \rho / \partial z$ is found to be proportional to the gravitational strength $g_{i}$ in fluid $i$. This density gradient it produced solely 

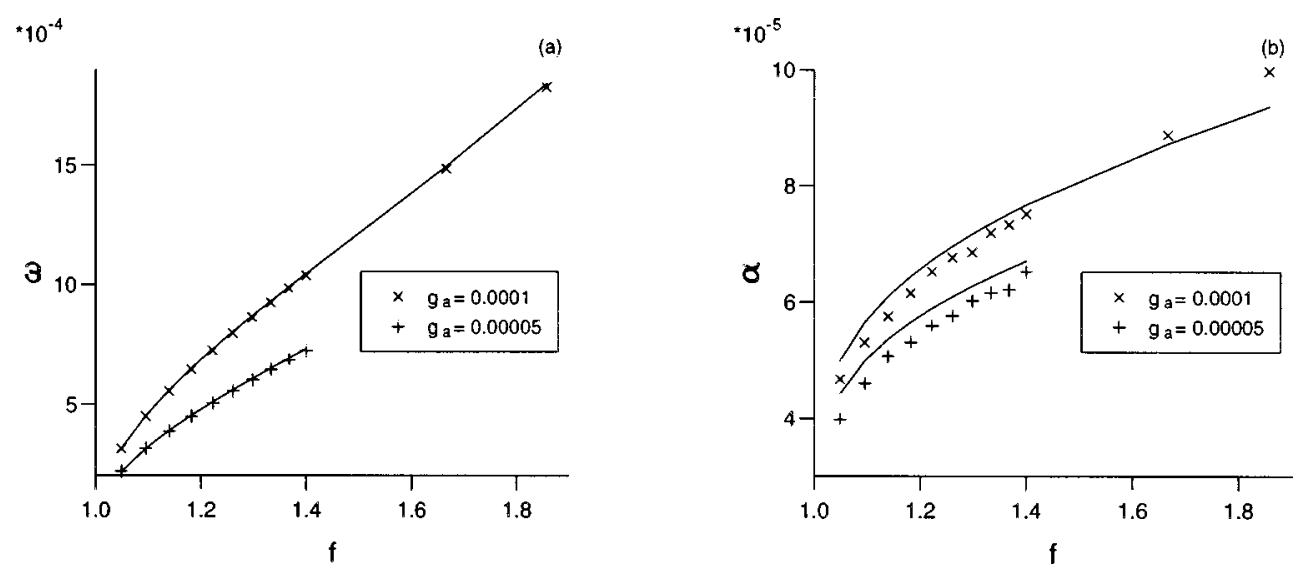

FIG. 8. (a) The frequency and (b) the damping rate as functions of the density ratio $f$ when $g_{a}$ is fixed, $\lambda=256$ and $\nu=0.05$. The solid lines are the theoretical curves.

by gravity so the potential density $\rho_{\text {pot }}$ is constant in each fluid. In Sec. $\mathrm{V}$ the equations describing interfacial wave motion were in terms of $f$, the ratio of the potential densities. If we consider two fluids with the same density, $\rho_{1}=\rho_{2}=\rho$, and apply gravity to fluid 1 with strength $g_{1}$ and to fluid 2 with strength $g_{2}$, then the ratio of the body forces, $\rho_{1} g_{1} / \rho_{2} g_{2}$, is $g_{1} / g_{2}$. This is the same as the ratio for two fluids with densities $\rho_{1}$ and $\rho_{2}=\left(g_{2} / g_{1}\right) \rho_{1}$ when gravity acts equally, with strength $g$, in each. Thus when we apply gravity with strength $g_{1}$ in fluid 1 and $g_{2}$ in fluid 2 it is equivalent to simulating two fluids with relative densities $g_{2} / g_{1}$ in a constant gravitational field. When the interface occurs close to the center of the grid $g$ is taken to be $\left(g_{1}\right.$ $\left.+g_{2}\right) / 2$.

\section{B. Surface tension}

Surface tension has not been considered in the analytic expressions despite it being inherent in the binary fluid model. Interfacial waves have a critical wavelength $\lambda_{c}$ given by $^{63}$

$$
\lambda_{c}=2 \pi \sqrt{\frac{\sigma}{\rho g(1-f)}},
$$

where $\sigma$ is the surface tension. At this wavelength gravity and surface tension are equally significant in determining the nature of the wave. Interfacial waves with wavelength $\lambda$ $>3 \lambda_{c}$ can be considered as being pure gravity waves while waves with $\lambda<\lambda_{c} / 3$ are surface tension driven capillary waves. The surface tension, $\sigma$, is related to the pressure change, $\Delta p$, across a bubble of radius $r$ of one fluid inside the other by Laplace's law:

$$
\Delta p=\frac{\sigma}{r} .
$$

The pressure difference was measured using a bubble of radius $r=20$ and the surface tension was found to be 3.4 $\times 10^{-4}$ for $\Lambda=1.1, \quad T=0.5, \quad \Gamma=0.1, \tau_{\Delta}=0.789$ and $\kappa$ $=0.001$. The smallest values of $g$ and $(1-f)$ used in the simulations are $1.05 \times 10^{-4}$ and 0.05 , respectively. This gives a maximum value of $3 \lambda_{c}=156$. With the exception of two simulations which were performed with $\lambda=128$ which is approximately 2.5 times the maximum value of $\lambda_{c}$, all the other simulations had $\lambda=256$ or 512 which are significantly larger than $3 \lambda_{c}$. Thus we conclude that the results obtained from the simulations should not be influenced by surface tension and so can be compared to the theoretical expressions of Sec. V.

\section{Wave frequency and damping rate}

The variation in the wave frequency and the damping rate with the other parameters was examined by performing simulations with different values of the model parameters. The results are shown in Figs. 8-12 where the solid lines are the theoretical values calculated from Eqs. (33), (34), (46), and (47). In Fig. 8 the parameter $g_{a}$ was fixed while $g_{b}$ was varied to give different values of $f$. The viscosity and the wavelength were fixed at 0.05 and 256 , respectively. The results are for $g_{a}=0.0001(X)$ and $g_{a}=0.00005(+)$. The results in Fig. 9 are for $\nu=0.05$ and $\lambda=256$ with $g=1.5$ $\times 10^{-4}(\times)$ and $g=5 \times 10^{-5}(+)$ where the density ratio $f$ is varied. Figure 10 has $g_{2}-g_{1}=5 \times 10^{-5}$. The viscosity and the wavelength were again fixed at 0.05 and 256 , respectively. Figure 11 is for $g_{1}=2.5 \times 10^{-4}, g_{2}=3.5 \times 10^{-4}$ $(\times)$ and $g_{1}=1.025 \times 10^{-4}, g_{2}=1.075 \times 10^{-4}(+)$. The wavelength is $\lambda=256$. Figure 12 is also for $g_{1}=2.5$ $\times 10^{-4}, g_{2}=3.5 \times 10^{-4}(\times)$ and $g_{1}=1.025 \times 10^{-4}, g_{2}$ $=1.075 \times 10^{-4}(+)$ when the viscosity is fixed at $\nu=0.05$.

In each case there is reasonable agreement between the results and the theory. When $\nu=0.05$ and $\lambda \geqslant 256$ the results found for the frequency are, in general, about $1 \%$ smaller than the theoretical predictions while the results for $\alpha$ are, on average, about $4 \%$ smaller. This is particularly noticeable in Fig. 10 where there is little variation in $\omega$ and $\alpha$ over the range of results. The results in Fig. 11(b) show a greater departure between the theory and the simulations for larger values of the viscosity; this can also be seen to a much lesser extent in 11(a). The results in Fig. 12 also show a larger difference between the theory and the simulations when $\lambda$ $=128(k=0.05)$. As before the difference is greater for the damping rate then it is for the frequency. Thus, for small $\nu$ 

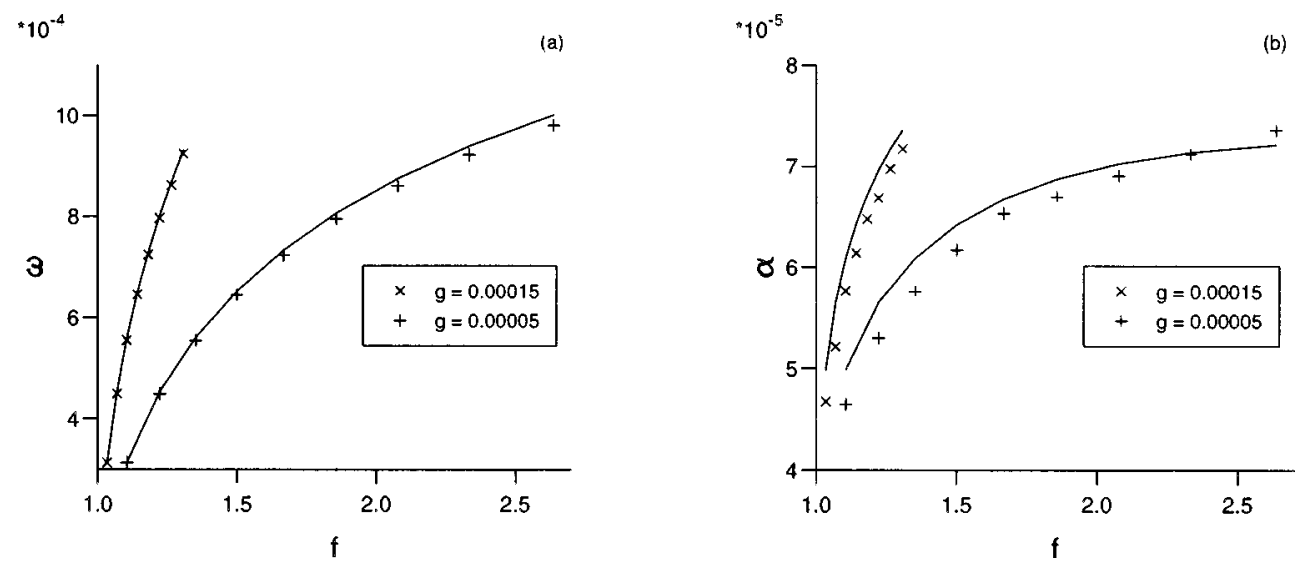

FIG. 9. (a) The frequency and (b) the damping rate as functions of the density ratio $f$ when the gravitational acceleration $g$ is fixed, $\lambda=256$ and $\nu=0.05$. The solid lines are the theoretical curves.
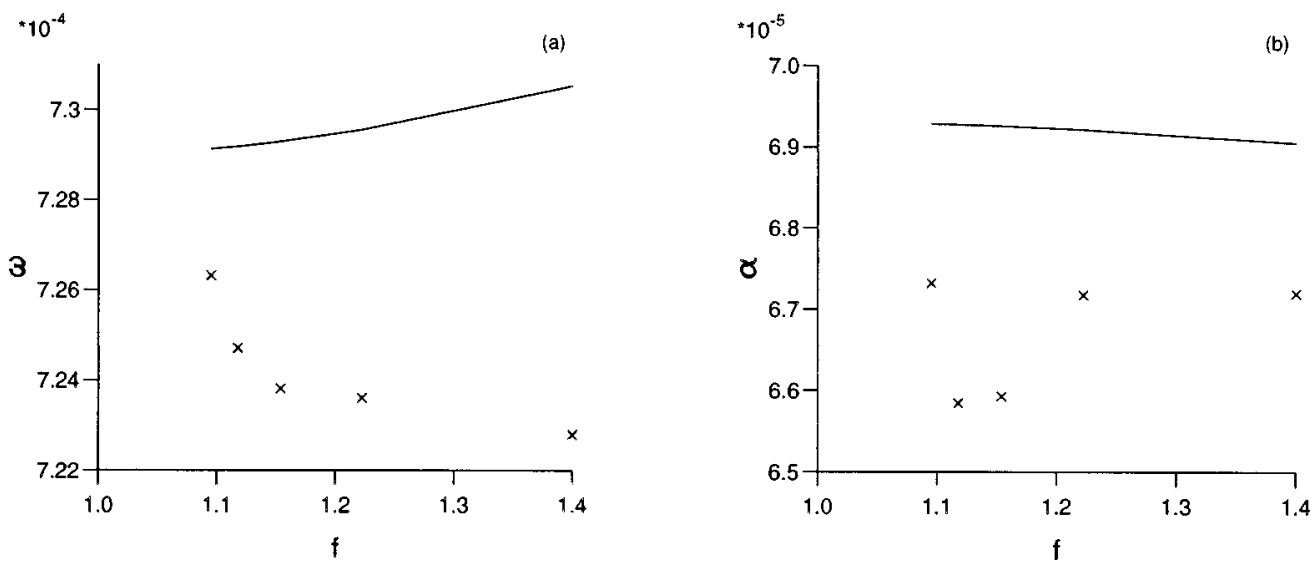

FIG. 10. (a) The frequency and (b) the damping rate as functions of the density ratio $f$ when the density difference is fixed by $g_{2}-g_{1}=5 \times 10^{-5}, \lambda$ $=256$ and $\nu=0.05$. The solid lines are the theoretical curves.
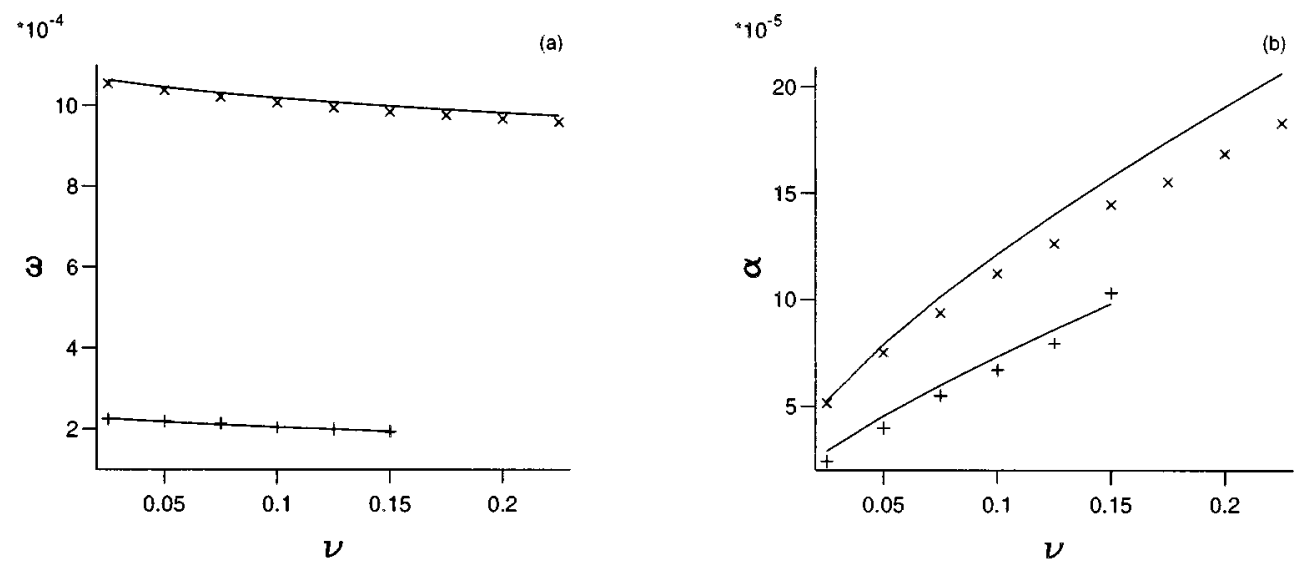

FIG. 11. (a) The frequency and (b) the damping rate as functions of the viscosity $\nu$. The results are for $g_{1}=2.5 \times 10^{-4}, g_{2}=3.5 \times 10^{-4}(\times)$ and $g_{1}$ $=1.025 \times 10^{-4}, g_{2}=1.075 \times 10^{-4}(+)$. The wavelength is $\lambda=256$. The solid lines are the theoretical curves. 

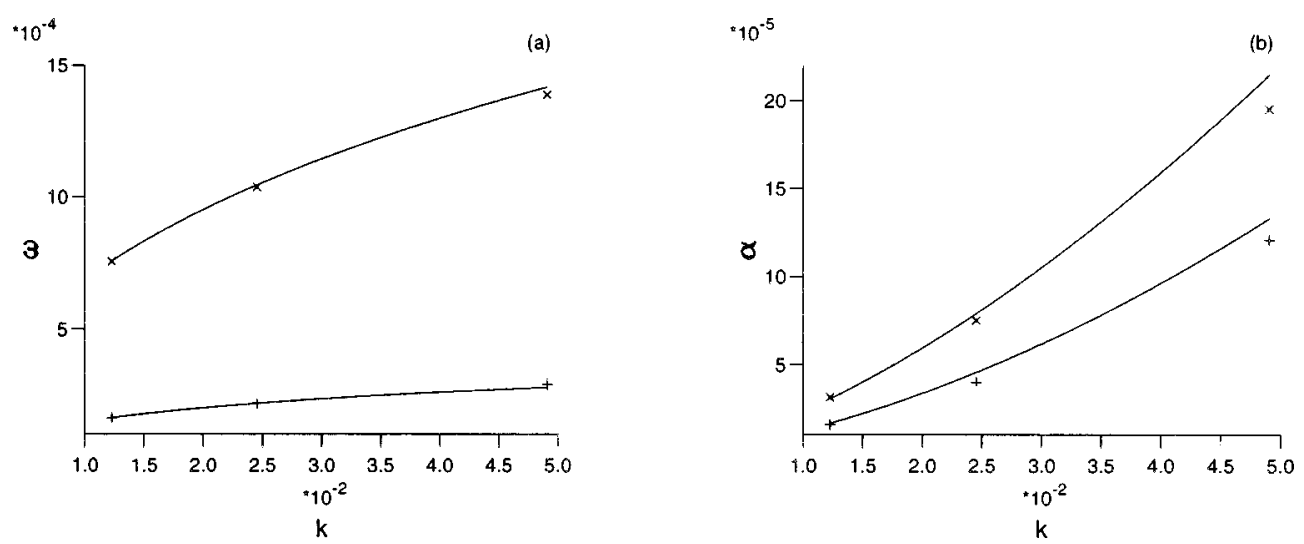

FIG. 12. (a) The frequency and (b) damping rate as functions of the wavenumber $k$. The results are for $g_{1}=2.5 \times 10^{-4}, g_{2}=3.5 \times 10^{-4}(\times)$ and $g_{1}$ $=1.025 \times 10^{-4}, g_{2}=1.075 \times 10^{-4}(+)$. The viscosity is $\nu=0.05$. The solid lines are the theoretical curves.

and small $k$, the regime where Eqs. (46) and (47) can be applied, there is good agreement between the results and the theory. For larger values of the viscosity and the wavenumber there are larger discrepancies, however, the theory is less accurate for these values since higher-order terms will become significant. The difference observed for $\omega$ and $\alpha$ between the computational results and the theory, where $\nu$ and $k$ are small, was found to be about $1 \%$ and $4 \%$, respectively. These are slightly larger than the $0.3 \%$ and $4 \%$ errors predicted in Sec. IV. Here, however, the fitted parameters are always smaller then their theoretical values. This might suggest that there is some bias in the fitting routine. No evidence of this was found when it was tested on data with a known frequency and damping rate. The discrepancy is more likely due to higher-order terms which are neglected in the theory and the ratio of the initial wave amplitude to the wavelength, $20 \sqrt{3} / 2$ :256 being slightly too large for the waves to be considered as truly linear. This ratio will be smaller at later times due to the high damping rate of the waves. The differences are nevertheless small and the comparison is good.

\section{Velocity measurements}

The fluid velocity is shown in Figs. $13-16$ at $t \simeq T / 4$ for the four waves shown in Table I. All four waves have $g$ in the range $1 \times 10^{-4} \rightarrow 5 \times 10^{-4}$. The value of $g$ affects both the magnitude and the shape of the velocity profile since $\omega$ is a function of $g$. Wave (1) has a relatively low viscosity and the lower fluid is significantly denser than the top fluid. Wave (2) also has a relatively low viscosity and the two fluids are of similar densities, varying by only $5 \%$. Wave (3) has the same viscosity as the first two waves but the density difference is considerable; the ratio of the densities is 1.86 . Wave (4) has the same density distribution as wave (1) but the viscosity is five times larger. For each wave the velocity is symmetric about and $x=\lambda / 2$. The velocities are not symmetric about $z=0$, although the contour plots are similar in both fluids. The difference is greatest when there is a large density difference corresponding to a large value of $f$. The magnitudes of the velocities are different for each wave. Wave (2) has velocities considerable smaller than the other waves. These lower velocities are shown in Fig. 14 where the contour lines are distorted slightly near the interface. This is due to small, spurious interface velocities which have been observed for the lattice Boltzmann model and which are due to the finite space and time steps. ${ }^{53}$ This is not observed for the other waves where the velocities are higher. For each wave the magnitudes of $u$ and $w$ are similar. The vertical velocity peaks at $z=0$ to a slightly higher value than the (a)

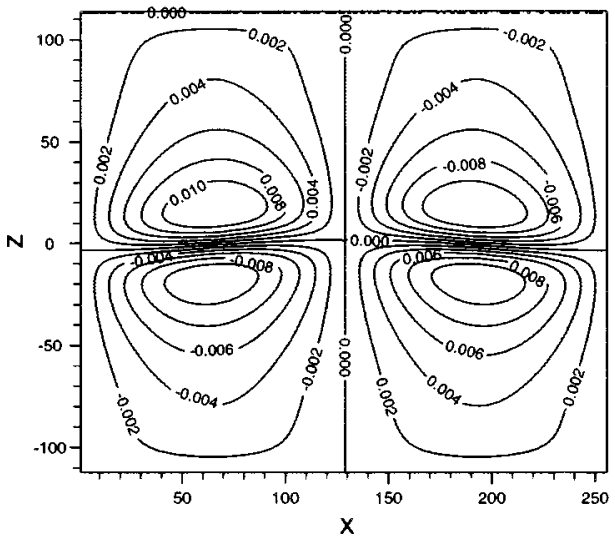

(b)

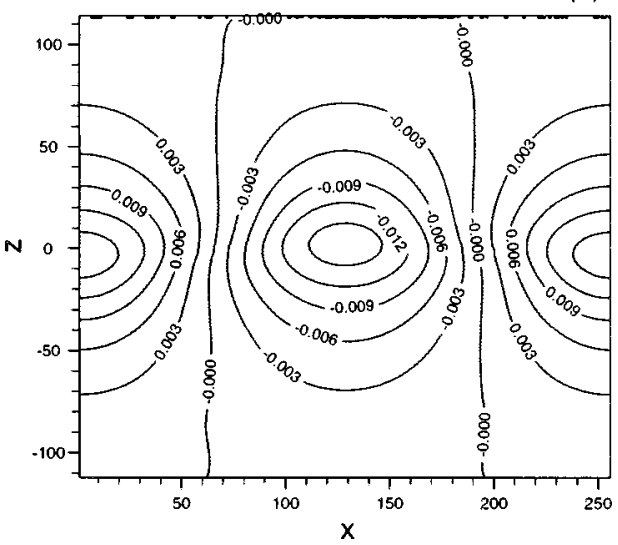

FIG. 13. (a) Horizontal and (b) vertical velocity contour plot for wave (1) with $\lambda=256, \nu=0.05, g=3 \times 10^{-4}$ and $f=1.4$ at $t \simeq T / 4$. 
(a)

(b)
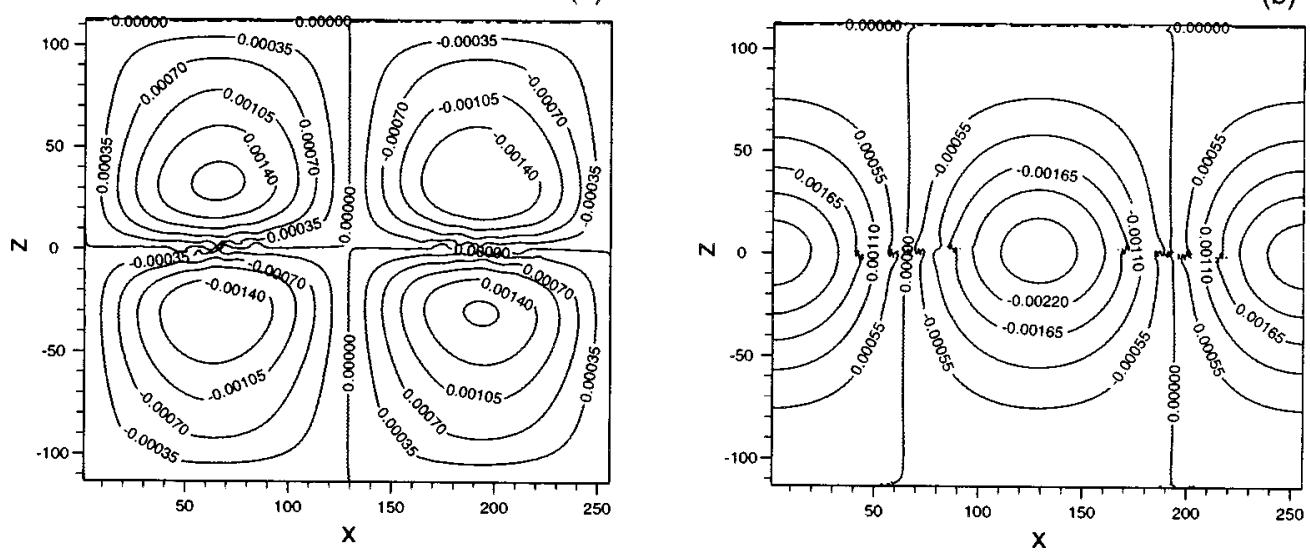

FIG. 14. (a) Horizontal and (b) vertical velocity contour plot for wave (2) with $\lambda=256, \nu=0.05, g=1.05 \times 10^{-4}$ and $f=1.05$ at $t \simeq T / 4$.

(a)
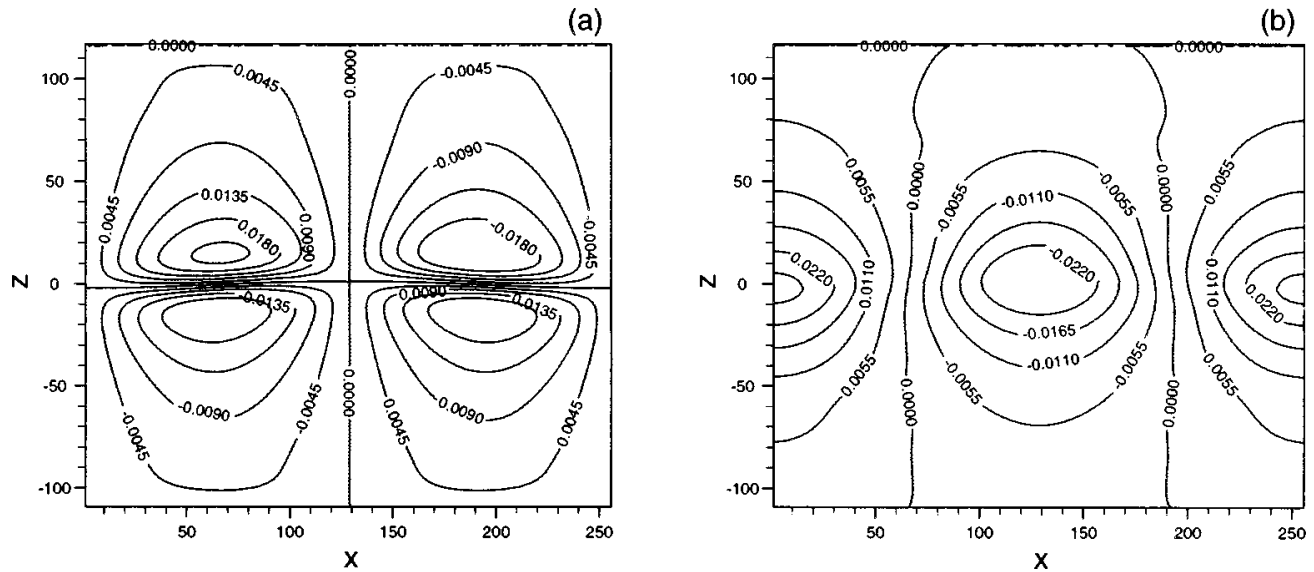

FIG. 15. (a) Horizontal and (b) vertical velocity contour plot for wave (3) with $\lambda=256, \nu=0.05, g=5 \times 10^{-4}$ and $f=1.86$ at $t \simeq T / 4$.

(a)

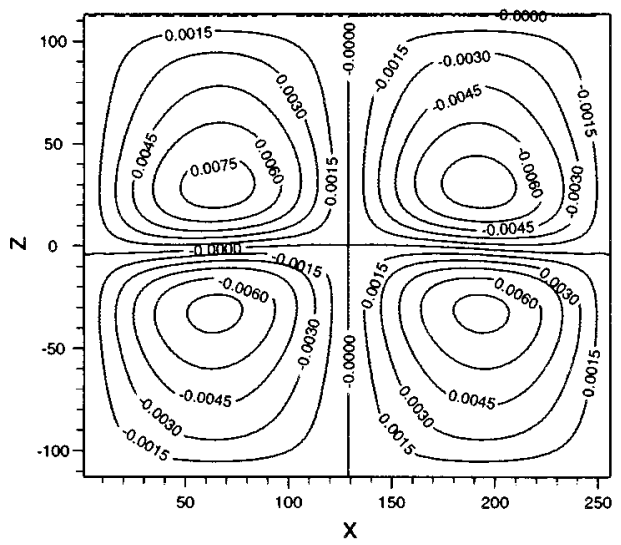

(b)

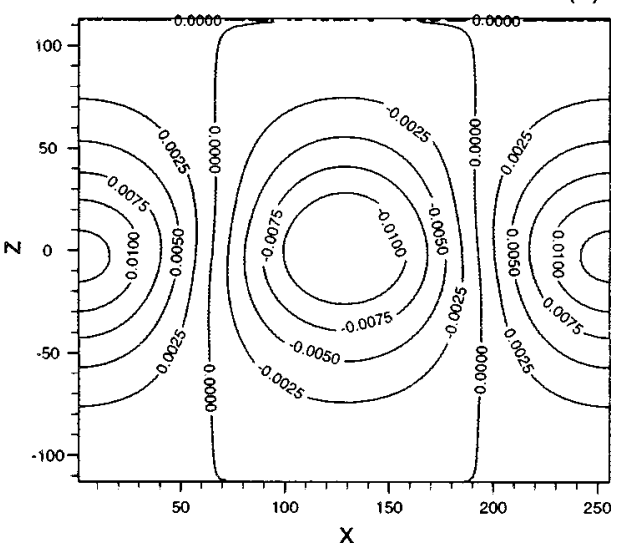

FIG. 16. (a) Horizontal and (b) vertical velocity contour plot for wave (4) with $\lambda=256, \nu=0.25, g=3 \times 10^{-4}$ and $f=1.4$ at $t \simeq T / 4$. 
TABLE I. The four waves in Figs. 13-16. All the values are measured in lattice units.

\begin{tabular}{cccrccr}
\hline \hline Wave & $\lambda$ & $\nu$ & \multicolumn{1}{c}{$g$} & $f$ & $\omega$ & $(\nu / \omega)^{1 / 2}$ \\
\hline$(1)$ & 256 & 0.05 & $3 \times 10^{-4}$ & 1.40 & $1.04 \times 10^{-3}$ & 6.94 \\
$(2)$ & 256 & 0.05 & $1.05 \times 10^{-4}$ & 1.05 & $2.19 \times 10^{-4}$ & 15.12 \\
$(3)$ & 256 & 0.05 & $5 \times 10^{-4}$ & 1.86 & $1.82 \times 10^{-3}$ & 5.23 \\
$(4)$ & 256 & 0.25 & $3 \times 10^{-4}$ & 1.40 & $9.59 \times 10^{-4}$ & 16.15 \\
\hline \hline
\end{tabular}

peak horizontal velocity, which occurs slightly above and below the interface. In wave (1) and wave (3) $u$ peaks close to the interface, the contours for the higher magnitudes have an elliptical appearance, the semi-major axis parallel to the interface. The contours for the lower magnitudes appear more triangular in shape with the base near the interface and the opposite angle considerably rounded. Waves (2) and (4), on the other hand, have $u$ peaking further from the interface. The high magnitude contours are much more circular and the lower magnitude contours are almost rectangular with curved corners. The lower magnitude contours for the vertical velocity are elliptical for all the waves, the semi-major axis being perpendicular to the interface. The higher magnitude vertical velocity contours for waves (2) and (4) are approximately circular while for waves (1) and (3) they are more elliptical in the same sense as the horizontal velocity.

\section{E. Velocity variation across a vertical cross-section}

The velocities found from the four waves are compared with the theoretical expressions, Eqs. (42)-(44), in Figs. $17-20$ where the horizontal velocity is measured at $x=\lambda / 4$, $t=T / 4$ and the vertical velocity at $x=\lambda / 2, t=T / 4$. This is where the magnitudes of the velocities are maximum. The solid lines are the theoretical values and the markers are the simulation results. For each wave there is a good agreement for both the magnitude and the shape of the velocity distributions. The poorest fit is for the horizontal velocity in wave (4) where the viscosity is at its largest. Despite the large viscosity $\epsilon$ in Eq. (35) is 0.05 which is still small so the theory should be applicable. For wave (4) the fit is good everywhere except outside the viscous interface region in the upper fluid. At its worst the discrepancy is about $1 \times 10^{-3}$, about $25 \%$, however the difference is generally much smaller, only a few percent. As predicted in Sec. V the viscosity has little effect on the shape of the vertical velocity distribution, the shape of the graphs in Figs. 17(b), 18(b), 19(b), and 20(b) all being similar. The difference in the velocity magnitude is due mainly to the different values of $\omega_{0} \propto[(f-1) g /(1+f)]^{1 / 2}$ as would be the case for inviscid waves. The effect of the viscosity is much more evident in the horizontal velocity profiles shown in Figs. 17(a), 18(a), 19(a), and 20(a). Waves (1) and (3) both show a definite boundary layer, in both fluids, in the region of the solid boundary (at $z \simeq \pm 110$ ). The influence of the boundary layer is only obvious within about 10 lattice-steps of the boundary. Waves (2) and (4) both have very low velocities close to the boundary and there is no noticeable change in the velocity profile. At the interface $(z=0)$ the effect of the viscosity can be seen readily in each of the waves. The magnitude of the horizontal velocity peaks some distance from the interface and then decreases steadily to zero at the interface. The distance of the peak from the interface is $\simeq 10$ lattice-steps for waves (1) and (3) and $\simeq 30$ lattice-steps for waves (2) and (4). This is consistent with the values of $(\nu / \omega)^{1 / 2}: 7,15,5$ and 16 given in Table I. For waves (1) and (3) $(\nu / \omega)^{1 / 2}$ has a similar value, about a third of the value for waves (2) and (4). At twice this distance magnitude of the rotational component of the velocity will be reduced by a factor of $e^{-2}$ to about $14 \%$ of its value at the interface/boundary and its effect will become negligible at greater distances.

The results in Fig. 21 shows the horizontal and the vertical velocity profile, respectively, at times $t \simeq T / 4,3 T / 4$, $5 T / 4,7 T / 4,9 T / 4$, and $11 T / 4$ for wave (1). The horizontal velocity is shown at $x=\lambda / 4$ and the vertical velocity at $x$ $=\lambda / 2$. The decrease in both the horizontal and the vertical velocity with time can be seen over the three periods of oscillation. This is expected at the rate $\exp (-\alpha t)$ since this is the rate at which $a$, the interface amplitude, is decaying and the velocities are proportional to $a$. There is also a lack of symmetry about $z=0$ which is particularly obvious in the horizontal velocity, see Fig. 21(a). This is expected since the ratio of the rotational velocities $C 1 / C 2$ is 1.4 here. (a)

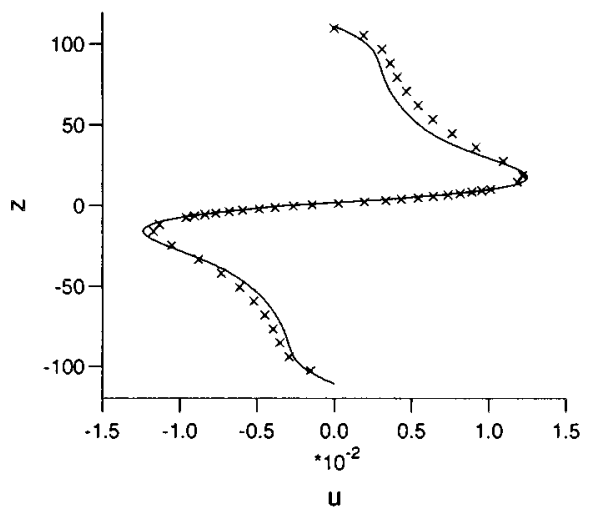

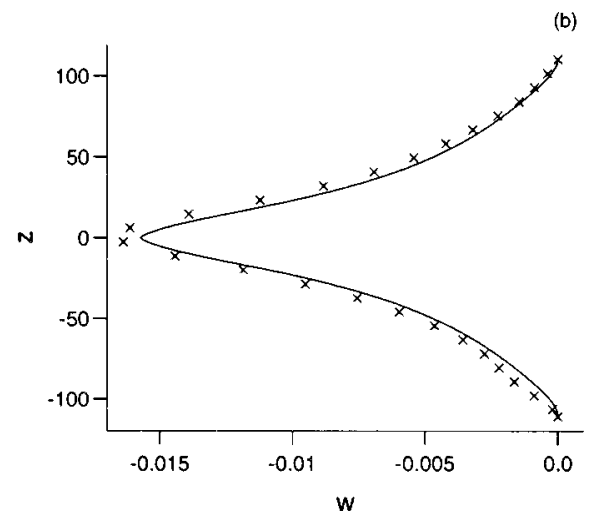

FIG. 17. (a) The horizontal velocity at $x=\lambda / 4$ and (b) the vertical velocity at $x=\lambda / 2$, both as functions of $z$ for wave (1) with $\lambda=256, \nu=0.05, g=3$ $\times 10^{-4}$ and $f=1.4$ at $t \simeq T / 4$. The solid lines are the theoretical curves. 

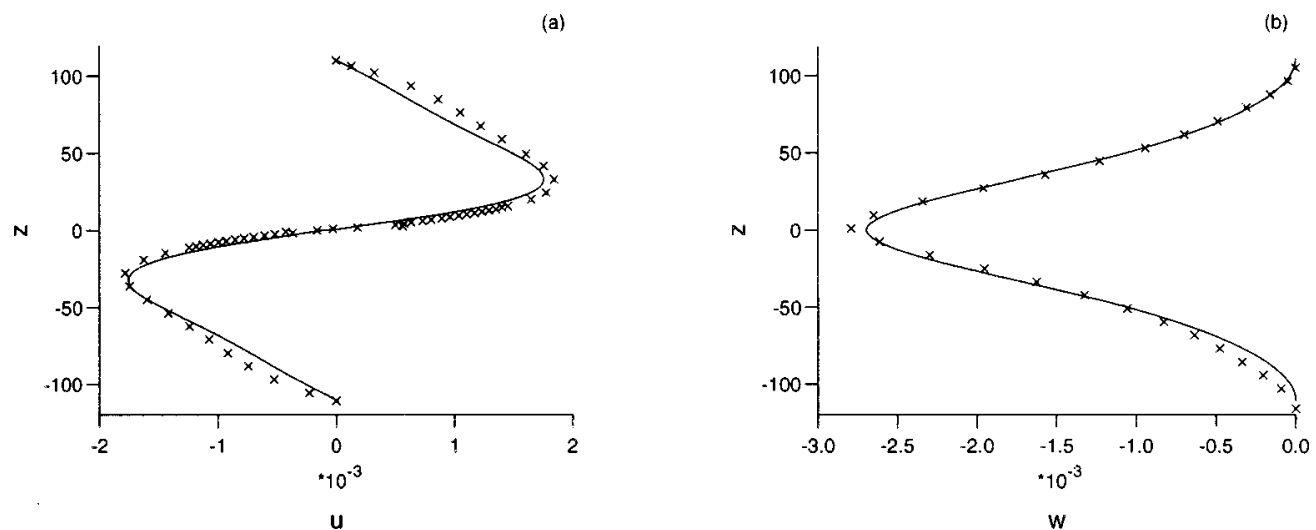

FIG. 18. (a) The horizontal velocity at $x=\lambda / 4$ and (b) the vertical velocity at $x=\lambda / 2$, both as functions of $z$ for wave (2) with $\lambda=256, \nu=0.05, g=1.05$ $\times 10^{-4}$ and $f=1.05$ at $t \simeq T / 4$. The solid lines are the theoretical curves.

\section{F. Velocity variation across a horizontal cross-section}

The velocity variation along horizontal cross-sections through the wave were also examined. This is shown in Figs. 22 and 23 for wave (1) at $t \simeq T / 4$. Figure 22 shows the horizontal and vertical velocities along cross-sections through the inviscid body of the fluid. Figure 23 shows the horizontal velocity along cross-sections through the viscous boundary layers near the solid boundaries and the interfacial region. Also plotted in the figures are sine and cosine curves with selected amplitudes; these are represented by the solid lines. The vertical velocity is very small within the boundary layer at the solid boundary.In the interfacial boundary layer the variation in $w$ is the same as that shown in Fig. 22(b).

The results in Fig. 22 show very good agreement with the sine and cosine curves. Thus the wave velocities are seen to be following the expected variation with horizontal distance. Note that the amplitude of the sine and cosine curves in Fig. 22 (and in Fig. 23) have been picked arbitrarily to give a good fit to the results and are not the amplitudes predicted by theory. This was done because any small deviation of the velocity profile from a sinusoidal variation would not be obvious if the simulation results were being compared to a sine curve with a different amplitude. The difference between the amplitude of the results and the amplitude predicted by Eqs. (42)-(44) is typically small and can be seen in
Fig. 17. The results in Figs. 23(a) show the comparison inside the boundary layer at the fixed boundaries. The fit here is not quite as good, the results varying slightly from the sine curves. Any small phase shift introduced by the rotational part of the velocity will be the same at all points and so should not affect the shape of the curves. The results in Fig. 23(b) show a poorer fit close to the interface. This is because the interface is not completely flat; see Fig. 13. For $z$ positive the results are closer to the interface, and hence smaller, at $x=\lambda / 2$ than they are at $x=0$ and $x=\lambda$. Conversely when $z$ is negative the results for $x=0$ and $x=\lambda$ are closer to the interface than the results for $x=\lambda / 2$. This distorts the results from the plotted sine curves.

\section{G. Boundary layer at the solid boundaries}

All of the waves simulated have been on a square grid with the interface near the center. This means that the depth of the two fluids $h_{1}$ and $h_{2}$ satisfy $k h_{i} \simeq \sqrt{3} \pi$. Thus $\tanh \left(k h_{i}\right) \simeq 0.99$ and both fluids can be considered as being deep. There can, however, be a finite velocity close to the solid boundaries and a boundary layer is formed, see for example Fig. 17. A wave with the same parameters as wave (1), in Table I, was initialized on a grid with twice the height and with the interface at the center so that $h_{1}=h_{2}=\sqrt{3} \lambda / 2$.
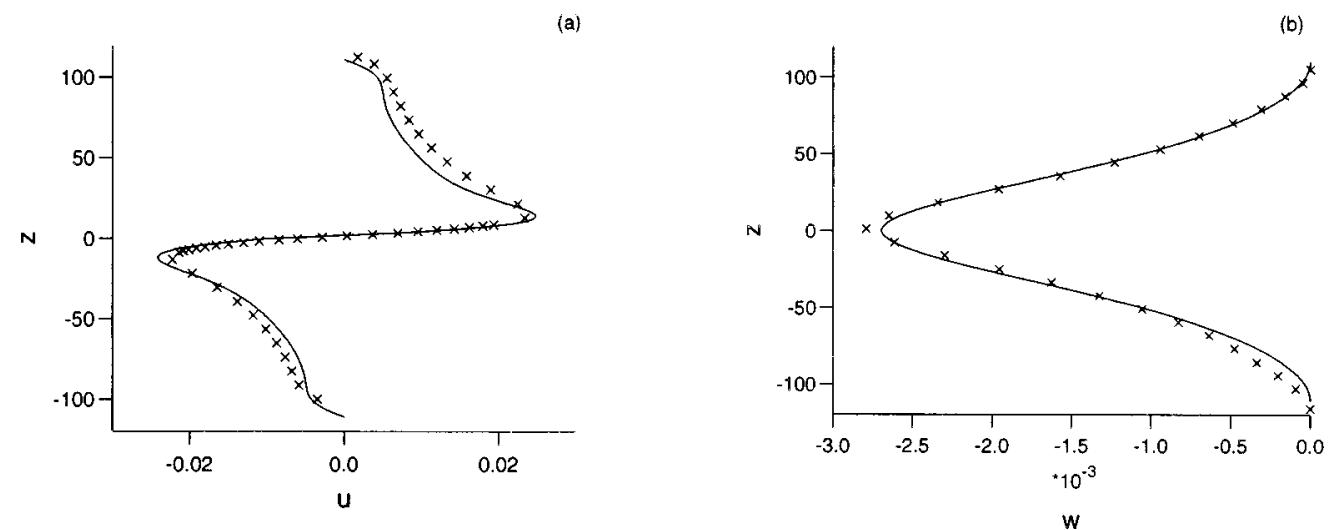

FIG. 19. (a) The horizontal velocity at $x=\lambda / 4$ and (b) the vertical velocity at $x=\lambda / 2$, both as functions of $z$ for wave (3) with $\lambda=256, \nu=0.05, g=5$ $\times 10^{-4}$ and $f=1.86$ at $t \simeq T / 4$. The solid lines are the theoretical curves. 

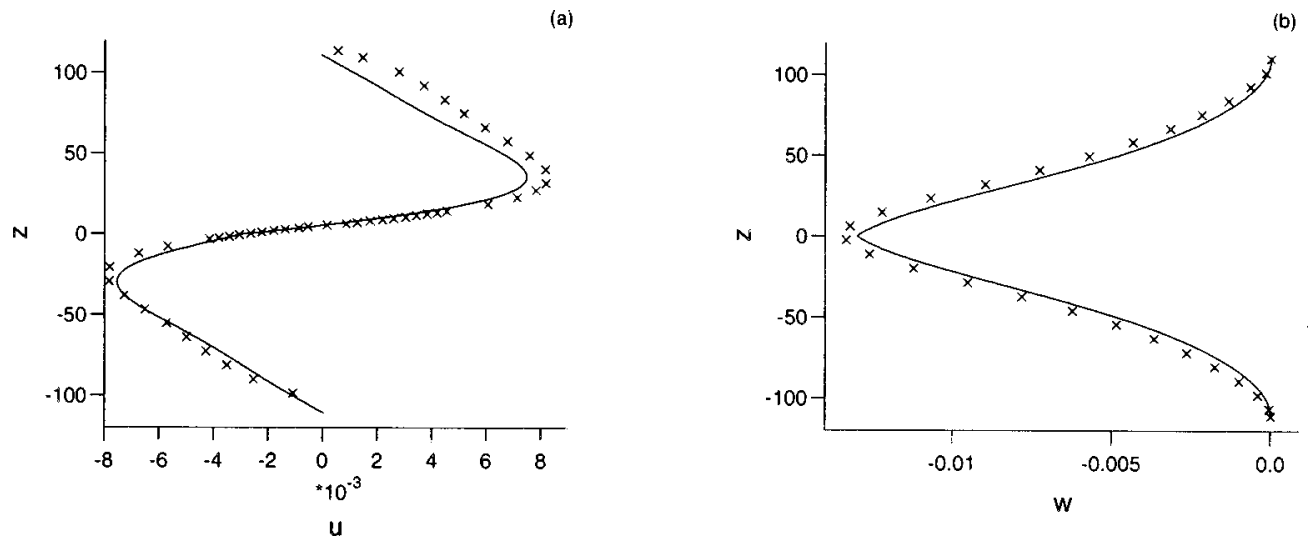

FIG. 20. (a) The horizontal velocity at $x=\lambda / 4$ and (b) the vertical velocity at $x=\lambda / 2$, both as functions of $z$ for wave (4) with $\lambda=256, \nu=0.25, g=3$ $\times 10^{-4}$ and $f=1.4$ at $t \simeq T / 4$. The solid lines are the theoretical curves.

The wave was allowed to evolve and the velocity profiles compared to those for wave (1). The velocity profiles for wave (1) and for the new wave on the larger grid are shown in Fig. 24. In the interface region the horizontal velocities, $u_{s}$ and $u_{l}$ of the wave on the small and large grid, respectively, are consistent. The horizontal velocity of the wave on the larger grid has become negligible within the inviscid region of the fluid and so there is no evidence of a boundary layer at the solid boundary. The boundary layer for the wave on the smaller grid can be observed, as before, and the horizontal
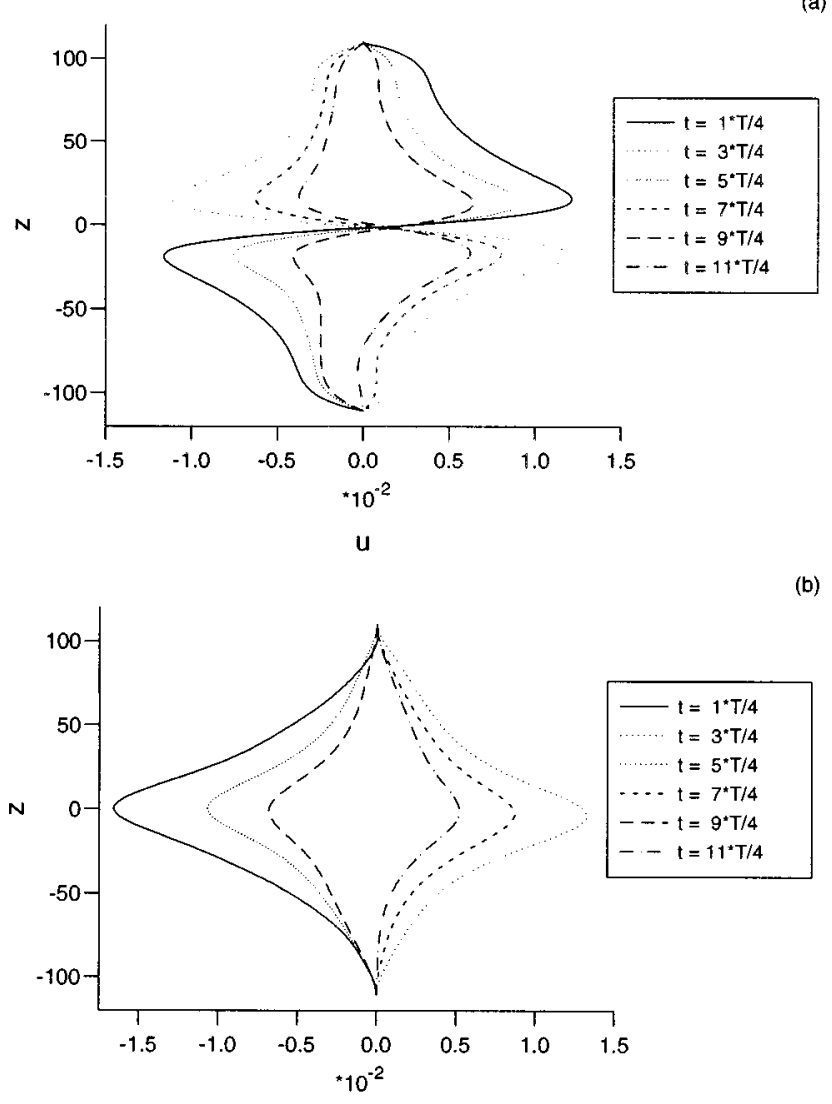

w

FIG. 21. (a) The horizontal velocity at $x=\lambda / 4$ and (b) the vertical velocity at $x=\lambda / 2$, both as functions of $z$ for wave (1) at multiples of $T / 4$. velocity is zero on the solid boundary, as expected. Away from the solid boundary $\left|u_{s}\right|$ increases rapidly and becomes slightly larger then $\left|u_{l}\right|$ outside its boundary layer. This is due partly to the well-known results that there is a region at the outer limit of the boundary layer where $U^{\prime}$ is small but not negligible and where $u_{0}$ and $U^{\prime}$ have the same sign. In this region $\left|u_{0}+U^{\prime}\right|>\left|u_{0}\right|$ and so the fluid velocity is slightly larger than it would be if the fluid was inviscid. The observed difference is, however, due mainly to the different values of $h_{i}$ for the two waves appearing in Eqs. (42) and (43). The results in Fig. 24(b) show that $w_{s}$ and $w_{l}$ are very similar everywhere except within the boundary layer of the smaller grid where $w_{s}$ approaches zero. The velocity $w_{l}$ is also small in this region and reaches zero slightly further from the interface and no boundary layer is observed at the solid boundary of the larger grid. In the region between $w_{l}$ approaching zero and $z= \pm 512 \sqrt{3} / 4$ we would expect $w_{l}$ to be zero. In fact, a small variation is observed. This is derived from a small vertical velocity which is produced at the interface at the beginning of the simulation when the density is very slightly reduced across the small interface region. The velocities produced are very small and are generally negligible, particularly when $\kappa=0.001$ and the interface is sharp. The frequency and damping rate were also found for the wave on the larger grid and were negligibly different from the results obtained when the smaller grid was used.

Thus we have seen that increasing the depth of both fluids has a negligible affect on the frequency and damping rate. The velocities are similar near the interface where they are largest. Away from the interface there is only a small difference in the velocities, mainly due to a boundary layer forming around the solid boundary.

\section{H. Peak horizontal velocity}

The results above all show that the magnitude of the horizontal velocity $|u|$ peaks above and below the interface within the boundary layer region. The height at which the peaks occur has been seen to depend roughly on the $(\nu / \omega)^{1 / 2}$. Here we look more closely at the dependence of $d$, the vertical distance between the two peaks, on the simulation parameters $f, \lambda$ and $\nu$. The values of $d$ was found from 

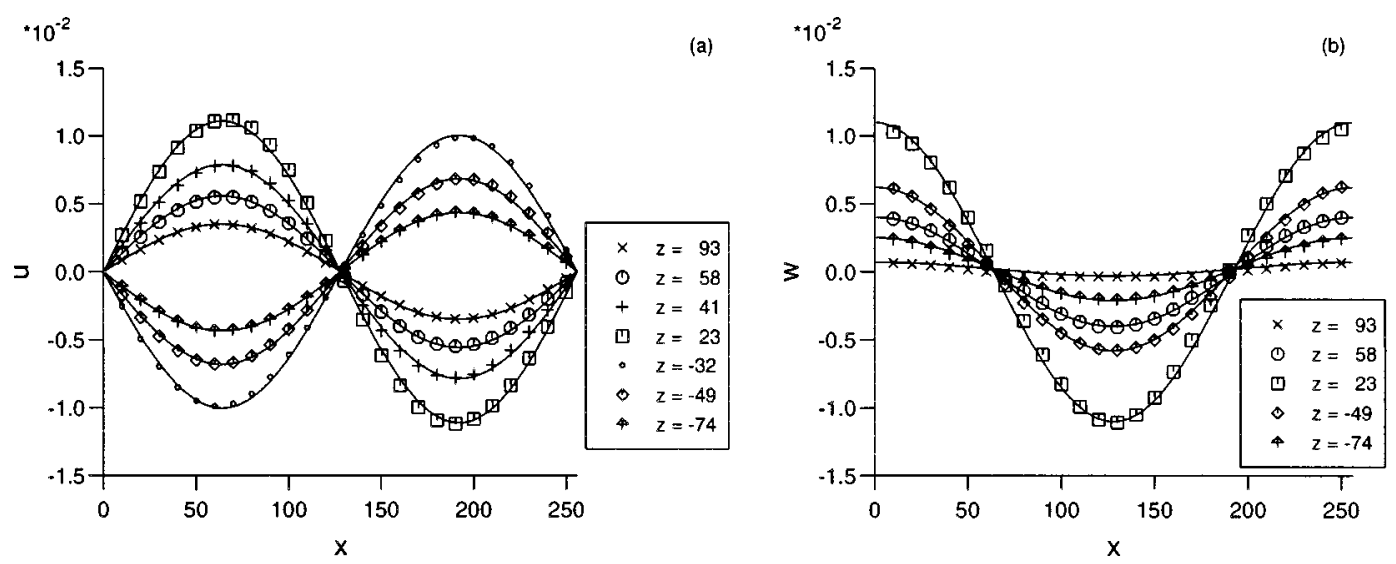

FIG. 22. (a) The horizontal and (b) the vertical velocities as functions of $x$ at $t \simeq T / 4$. The results are for wave (1) at different heights $z$ within the inviscid body of the wave. The solid lines are sine and cosine curves with an appropriate amplitude.
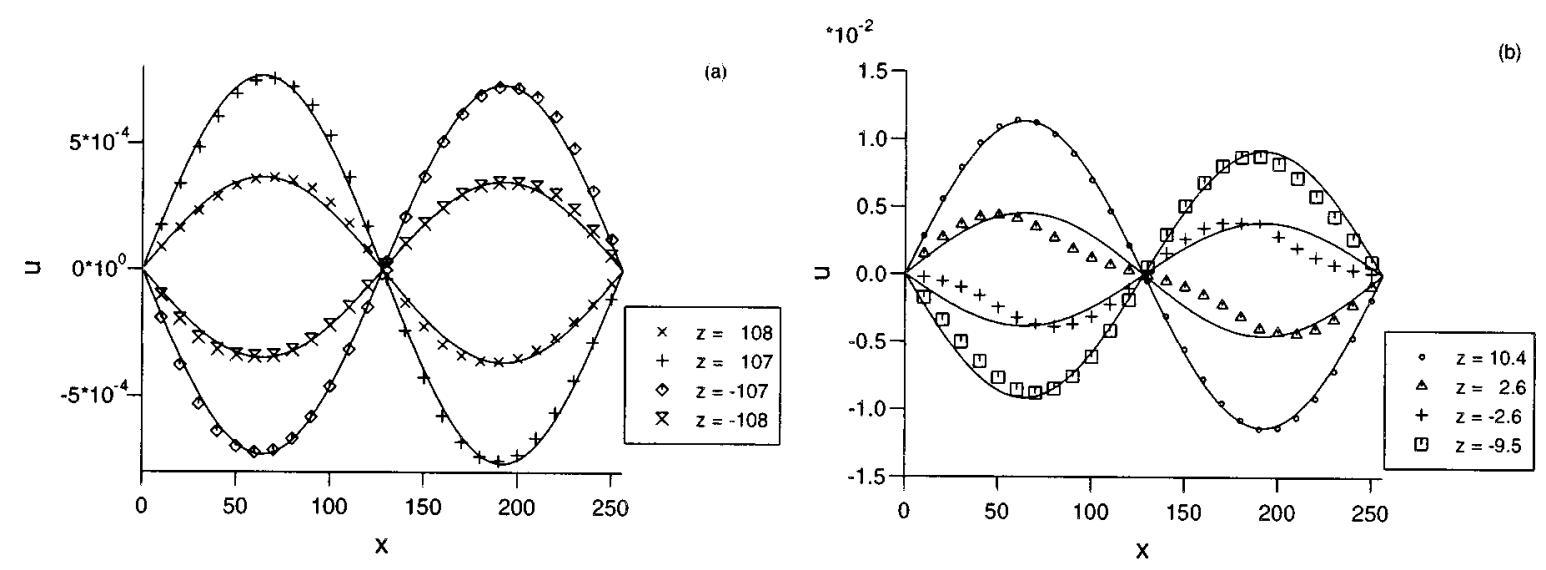

FIG. 23. The horizontal velocity $u$ as a function of $x$ at $t \simeq T / 4$. The results are for wave (1) at different heights $z$ (a) within the viscous boundary layer at the solid boundaries and (b) at the interface. The solid lines are sine curves with an appropriate amplitude.

(a)

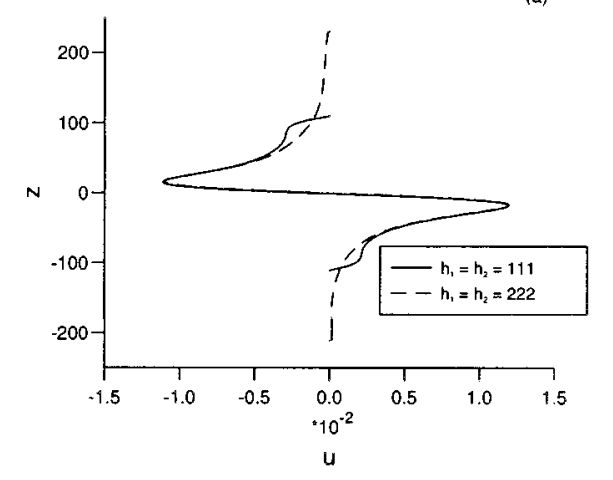

(b)

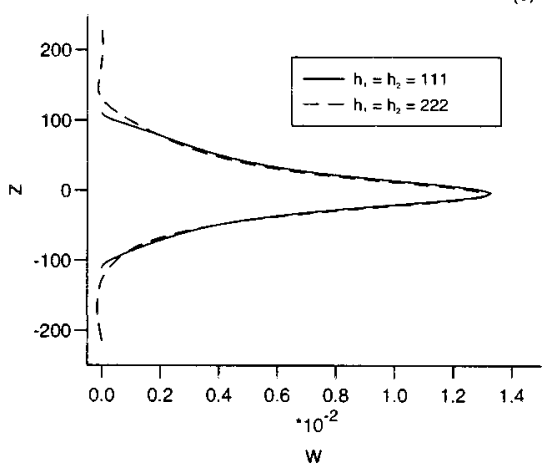

FIG. 24. (a) The horizontal velocity $u$ at $x=\lambda / 4$ and (b) the vertical velocity $w$ at $x=\lambda / 2$, both as a function of $z$ for wave (1) using two different grid sizes. The results are shown at $t \simeq 3 T / 4$. 

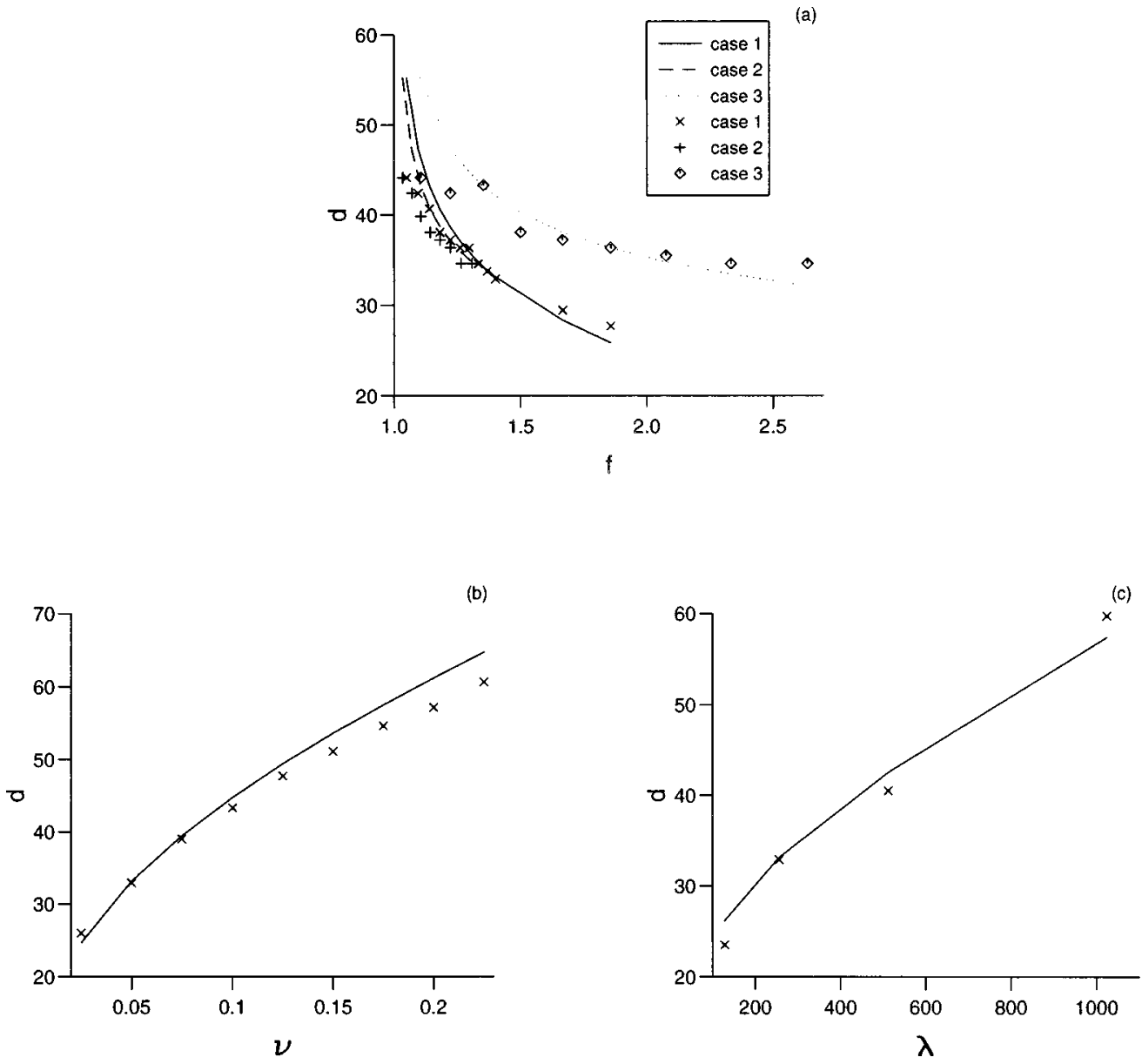

FIG. 25. The vertical separation $d$ of the horizontal velocity peaks (a) as a function of $f$ for three cases. Case (1) has $g_{a}=0.0001, \nu=0.05, \lambda=256$ and $g_{b}$ varied. Case (2) has $g=1.5 \times 10^{-4}, \nu=0.05$ and $\lambda=256$. The values of $g_{1}$ and $g_{2}$ are varied to give different values of $f$ while keeping $g$ fixed. Case (3) has $g_{2}-g_{1}=5 \times 10^{-5}, \nu=0.05$ and $\lambda=256$. (b) As a function of the viscosity $\nu$ when $g_{1}=2.5 \times 10^{-4}, g_{2}=3.5 \times 10^{-4}, f=1.4$ and $\lambda=256$. (c) As a function of the wavelength $\lambda$ when $g_{1}=2.5 \times 10^{-4}, g_{2}=3.5 \times 10^{-4}, f=1.4$ and $\nu=0.005$. The marks represent the simulated data and the lines the theoretical separation.

the simulations when $t=T / 4$ at $x=\lambda / 4$ and are correct to within one lattice-step. The theoretical values were obtained by finding the zeros of $d u_{1} / d z$ and $d u_{2} / d z$ (Ref. 64) where $u_{1}$ and $u_{2}$ are given in Eqs. (42) and (43). The results are shown in Fig. 25. The results in Fig. 25(a) are for three cases. Case (1) has $g_{a}=0.0001, \nu=0.05, \lambda=256$ and $g_{b}$ varied. Case (2) has $g=1.5 \times 10^{-4}, \nu=0.05$ and $\lambda=256$. The values of $g_{1}$ and $g_{2}$ are varied to give different values of $f$ while keeping $g$ fixed. Case (3) has $g_{2}-g_{1}=5 \times 10^{-5}, \nu=0.05$, and $\lambda=256$. There is good agreement with the theory except for the lowest values of $f$ where the theory predicts a value for $d$ which is significantly larger than the one measured in the simulations. When the boundary layer is large compared to the wavelength the assumptions made in deriving the velocity expressions are no longer valid. The results in Fig. 25(b) show the variation in $d$ for different values of the viscosity when $g_{1}=2.5 \times 10^{-4}, g_{2}=3.5 \times 10^{-4}, f=1.4$ and $\lambda=256$. Here the simulation results also show good agreement with the theory except at the highest values of the viscosity where, again, the size of the boundary layer is becoming significant compared to the wavelength. The results here suggest that, provided the size of the boundary layer (taken here to be $d / 2$ ) is less than $\lambda / 10$, there is good agreement between the expressions found from the theory and the simulation results. Figure 25(c) shows the value of $d$ for different wavelengths when $g_{1}=2.5 \times 10^{-4}, g_{2}=3.5 \times 10^{-4}, f=1.4$, and $\nu=0.005$. A square grid was used here so $h_{1}=h_{2}$ $=\sqrt{3} \lambda / 2$ and $d \ll \lambda$ for each wavelength. Again a good fit is observed between the simulation points and the theory. The size of the boundary layer about the interface is seen, in Fig. 25(c), to increase with the wavelength when all other parameters are unchanged. Despite this increase in the size of the boundary layer the dimensionless size of the boundary layer $d / \lambda$, which is shown in Fig. 26(a), decreases with increasing $\lambda$. This is as expected since as $\lambda$, and hence the Reynolds number of the wave, is increased, it is expected that the viscous effects become less significant. The profile of the vertical velocity at $t \simeq T / 4, x=\lambda / 2$ is also shown in Fig. 26(b) for the same waves. The viscosity has less effect on the vertical velocity and all the profiles have the same shape with a different amplitude. There is some small variations in $w$ which can be observed near the solid boundaries of the waves with the larger wavelengths. These are similar to those observed in Fig. 24(b) and are also due to the initial separation of the interface. It is also possible that there may be a 

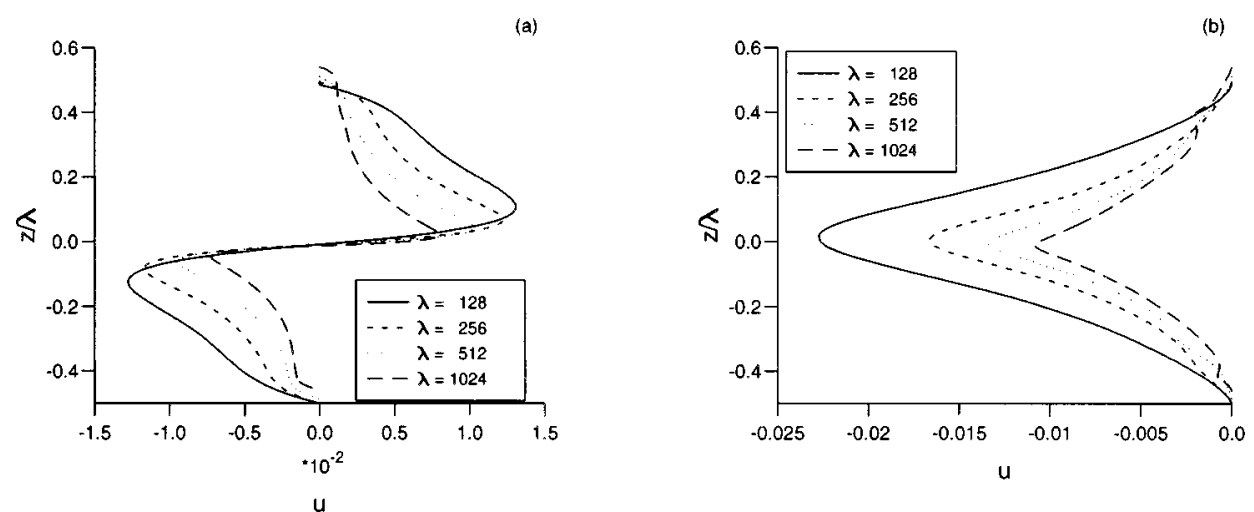

FIG. 26. (a) The horizontal velocity profile, at $x=\lambda / 4$ and (b) the vertical velocity at $x=\lambda / 2$, both as a function of the dimensionless parameter $z / \lambda$ at $t$ $\simeq T / 4$. This is for waves with $g_{1}=2.5 \times 10^{-4}, g_{2}=2.5 \times 10^{-4}, f=1.4$ and $\nu=0.005$.

small residual oscillation from the initial settling of the density gradient. However, every care was taken to ensure this had damped out before the simulations were performed.

\section{RELATING THE SIMULATIONS TO PHYSICAL PROBLEMS}

All the results presented here are in lattice units, the unit of length is the lattice-step and the unit of time is the timestep. When comparing the results of the simulations to real physical situations we must compare dimensionless quantities. Here we consider the values of these dimensionless parameters calculated from the results presented in Sec. VI. In each simulation both the fluids can be considered as being deep since $\tanh (k h)=0.99$. We also consider all the waves to be linear since the initial amplitude, $A=\sqrt{3} / 2 \times 20$, is generally small compared to the wavelengths used, $\lambda=128,256$, 512. When the smallest wavelength is used the wave is strongly damped so any initial nonlinear deviations will quickly become negligible. The Reynolds number defined $\operatorname{Re} \equiv \omega A \lambda / \nu$ ranges between $\operatorname{Re} \approx 10 \rightarrow \operatorname{Re} \approx 1000$ for the range of parameters used here. The internal Froude number defined as $F \equiv \omega A\left(k / g^{\prime}\right)^{1 / 2}$ is given by $F=0.85,0.43$ and 0.21 for the three wavelengths used. The final dimensionless parameter which describes the simulations is $f$, the ratio of the density in the two fluids. The simulations presented here were in the range $f=1.05 \rightarrow f=1.86$.

The application of this technique is not limited to the range of dimensionless parameters quantified above. The depth of either or both fluids can be altered during the initialization by changing the size of the grid and/or the positions of the boundaries and the interface. The initial wave amplitude can also be altered during the initialization process. Here we restricted ourselves to linear waves because we wanted to test the model by simulating a problem with a well-known solution. In principle the technique should be equally applicable to simulating steep waves although this has still to be investigated. The Reynolds number of the wave can be increased by increasing $g^{\prime}, \lambda$ and $A$ and by decreasing $\nu$. Increasing the wavelength requires an increase in the grid size and hence an increase in the computer time required, the other variables can be changed without altering the speed of the simulation (although clearly if the wave period is increased the simulation must be run for longer to simulate the same number of periods). The internal Froude number for linear waves in deep water is simply given by $F=2 \pi A / \lambda$ through the dispersion relation and so only depends on the wavelength. A much wider range of Froude numbers could be obtained if the variations in fluid depth and wave steepness discussed above were implemented. The density ratio of the two fluids can also be varied out with the range used here although in practice you would not expect a larger density ratio.

\section{CONCLUSION}

In this paper we have considered the lattice Boltzmann approach to fluid simulation. We have seen that the method can be applied to model a binary fluid mixture and that a body force can be introduced in such a way that the gravitational force produced in each fluid is different. We have shown that such a model can be applied to the study of interfacial standing waves. We have also considered any errors in the measurements made from the simulation which are typically small. Measurements made from lattice Boltzmann simulations of linear interfacial gravity waves in deep water have been compared with the standard theory. Using the initialization method it is possible to initialize a standing wave at the interface between two immiscible fluids of different densities. This can be done for a chosen wavelength and wave amplitude using the natural density gradient, correct for the values of $g_{i}$ being used. This method of initialization does not require that any velocities or a frequency are imposed on the wave. This means that any measured quantity has arisen solely from the wave motion. Many standing waves have been simulated for different values of the wavelength $\lambda$, the gravitational strengths $g_{1}$ and $g_{2}$, the density ratio $f$, and the viscosity $\nu$ which is varied through the relaxation time $\tau_{\rho}$. The measured frequencies, damping rates and velocities were compared to the theoretical predictions. All the results for the frequency were found to be in good agreement with the theory; the damping rate was also seen to be in good agreement with the theory over a range of values where the theory is applicable. The measured velocities were also seen to agree well with theory. The dimensionless parameters describing the simulated waves were also exam- 
ined. The waves were all linear waves in deep water with a Reynolds number between 10 and 1000 and a Froude number between 0.213 and 0.85 .

The simulations presented here have the advantage over previous numerical investigations ${ }^{25-31}$ that both the fluids have a fully viscous nature. This is a factor in real ocean waves. Although viscosity has been incorporated in some internal wave theories it has not been considered in numerical models. The method is generally applicable to deepwater, shallow-water and intermediate regimes. In the numerical studies discussed above $\mathrm{e}^{25-31}$ an equation, or set of equations, has been derived subject to different assumptions about the nature of the fluid, the wave and the order of accuracy required. This equation is then solved numerically to find the required quantity, for example the interface shape. Here, in common with the other techniques, we assume that the fluid is incompressible. We also assume that the fluid velocities are small with respect to the speed of sound. This is required since the lattice Boltzmann model satisfies the incompressible Navier-Stokes equation up to second order in the fluid velocity. The low Mach requirement can be satisfied by a suitable selection of the parameters regardless of the ratio of the wave amplitude to the wavelength. Thus this assumption is different from the assumption, usually made in interfacial wave simulations, that this ratio is in some sense small. Here the wave height is the position of the interface between the two fluids which is not dependent on any additional assumptions. There are no difficulties associated with tracking the interface as there can be when other methods are implemented. ${ }^{65}$ This suggests that the lattice Boltzmann model which has been shown to be correct for linear interfacial waves has significant potential for studying nonlinear and breaking waves.

Another area of interest which can be investigated using this model is the interaction between internal waves and variable topography. ${ }^{20,66,67}$ While the boundary conditions applied here can not be applied to an irregular boundary, simple reflection boundary conditions can, ${ }^{68}$ enabling complex bed boundaries to be studied without the need to consider complex numerical boundary conditions.

In conclusion, we have considered a lattice Boltzmann model for simulating interfacial waves between immiscible, viscous fluids. The model has been shown to correctly simulate linear waves and shows considerable potential for tackling more complex situations.

\section{ACKNOWLEDGMENTS}

The authors would like to thank the Edinburgh Parallel Computing Center (EPCC) for the generous amount of time made available on their Connection Machine CM-200.

${ }^{1}$ D. Halpern, "Observations on short-period internal waves in Massachusetts Bay," J. Mar. Res. 29, 116 (1971).

${ }^{2}$ L. R. Haury, M. G. Briscoe, and M. H. Orr, "Tidally generated internal wave packets in Massachusetts Bay USA.: Preliminary physical and biological results," Nature 278, 312 (1979).

${ }^{3}$ A. R. Osborne and T. L. Burch, "Internal solitons in the Andaman Sea," Science 208, 451 (1980).

${ }^{4}$ D. M. Farmer and J. D. Smith, "Tidal interaction of stratified flow with a sill in Knight Inlet," Deep Sea Res. 27A, 239 (1980).
${ }^{5} \mathrm{C}$. H. Mortimer, "Water movements in lakes during summer stratification; evidence from the distribution of temperature in Windermere," Philos. Trans. R. Soc. London, Ser. B 236, 355 (1952).

${ }^{6} \mathrm{~S}$. A. Thorpe, "Near-resonant forcing in a shallow two-layer fluid: a model for the internal surge in Loch Ness?," J. Fluid Mech. 63, 509 (1974).

${ }^{7}$ D. R. Christie, K. J. Muirhead, and A. L. Hales, "'On solitary waves in the atmosphere,' J. Atmos. Sci. 35, 805 (1978).

${ }^{8}$ R. S. Scorer and M. Wilkinson, "Waves in the lee of an isolated hill," $\mathrm{Q}$. J. R. Meteorol. Soc. 82, 419 (1956).

${ }^{9}$ E. E. Gossard and W. H. Hooke, Waves in the Atmosphere (Elsevier, Amsterdam, 1975).

${ }^{10}$ F. Nansen, Farthest North (Archibald Constable, Westminster, 1897).

${ }^{11}$ V. W. Ekman, "On dead-water," in The Norwegian North-Polar Expedition, 1893-1896: Scientific Results, edited by F. Nansen (Longmans, Green, New York, 1906), Vol. 5, pp. 1-152.

${ }^{12} \mathrm{C}$. Staquet and J. Sommeria, "Internal waves, turbulence and mixing in stratified flows: A report on Euromech Colloquium 339," J. Fluid Mech. 314, 349 (1996).

${ }^{13}$ A. Osborne, T. Burch, and R. Scarlet, "The influence of internal waves on deep water drilling,"’ J. Petroleum Technol. 30, 1497 (1978).

${ }^{14} \emptyset$. Arnsten, "Uniform stratified flow interaction with a submerged horizontal cylinder- a revisit of 'deadwater' in a Norwegian Fjord," Ph.D. thesis, University of Trondheim, Norwegian Institute of Technology, 1990.

${ }^{15}$ W. J. Harrison, "The influence of viscosity on the oscillations of superposed fluids,"' Proc. London Math. Soc. 2 6, 396 (1908).

${ }^{16} \mathrm{~B}$. Johns, "A boundary layer method for the determination of the viscous damping of small amplitude gravity waves,"' Q. J. Mech. Appl. Math. 21, 93 (1968).

${ }^{17}$ S. A. Thorpe, "On standing internal gravity waves of finite amplitude," J. Fluid Mech. 32, 489 (1968).

${ }^{18} \mathrm{~S}$. A. Thorpe, "On the shape of progressive internal waves," Philos. Trans. R. Soc. London, Ser. A 263, 563 (1968).

${ }^{19}$ J. Y. Holyer, "Large amplitude progressive interfacial waves," J. Fluid Mech. 93, 433 (1979).

${ }^{20}$ K. R. Helfrich and W. K. Melville, "On long nonlinear internal waves over slope-shelf topography," J. Fluid Mech. 167, 285 (1986).

${ }^{21} \mathrm{P}$. Christodoulides and F. Dias, "Stability of capillary-gravity interfacial waves between two bounded fluids,"' Phys. Fluids 7, 3013 (1995).

${ }^{22} \mathrm{~F}$. Dias and T. J. Bridges, "Geometric aspects of spatially periodic interfacial waves," Stud. Appl. Math. 93, 93 (1994).

${ }^{23}$ C. G. Koop and G. Butler, "An investigation of internal solitary waves in a two-fluid system," J. Fluid Mech. 112, 225 (1981).

${ }^{24} \mathrm{H}$. Michallet and E. Barthélemy, "Ultrasonic probes and data processing to study interfacial solitary waves," Exp. Fluids 22, 380 (1997).

${ }^{25}$ J.-M. Vanden-Broeck, "Numerical calculation of gravity-capillary interfacial waves of finite amplitude,"' Phys. Fluids 23, 1723 (1980).

${ }^{26}$ R. E. L. Turner and J.-M. Vanden-Broeck, "The limiting configuration of interfacial gravity waves," Phys. Fluids 29, 372 (1986).

${ }^{27}$ D. I. Meiron and P. G. Saffman, "'Overhanging interfacial gravity waves of large amplitude," J. Fluid Mech. 129, 213 (1983).

${ }^{28}$ A. J. Roberts, "A stable and accurate numerical method to calculate the motion of a sharp interface between fluids," IMA J. Appl. Math. 31, 13 (1983).

${ }^{29}$ D. I. Pullin and R. H. J. Grimshaw, "Nonlinear interfacial progressive waves near a boundary in a Boussinesq fluid," Phys. Fluids 26, 897 (1983).

${ }^{30}$ R. H. D. Grimshaw and D. I. Pullin, "Extreme interfacial waves," Phys. Fluids 29, 2802 (1986).

${ }^{31}$ D. I. Pullin and R. H. J. Grimshaw, "Interfacial progressive gravity waves in a two-layer shear flow,' Phys. Fluids 26, 1731 (1983).

${ }^{32}$ H. Sandstrom and J. A. Elliott, "Internal tide and solitons in the Scotian Shelf: A nutrient pump at work," J. Geophys. Res. 89, 6415 (1984).

${ }^{33} \mathrm{~N}$. S. Martys and H. Chen, "Simulation of multicomponent fluids in complex three-dimensional geometries by the lattice Boltzmann method," Phys. Rev. E 53, 743 (1996).

${ }^{34}$ J. M. Buick and C. A. Greated, "Gravity in a lattice Boltzmann model," submitted to J. Stat. Phys.

${ }^{35}$ W. R. Osborn, E. Orlandini, M. R. Swift, J. M. Yeomans, and J. R. Banavar, "Lattice Boltzmann study of hydrodynamic spinodal decomposition," Phys. Rev. Lett. 75, 4031 (1995).

${ }^{36}$ A. J. Wagner and J. M. Yeomans, "Effect of shear on droplets in a binary mixture," Int. J. Mod. Phys. C 8, 773 (1997). 
${ }^{37}$ J. Buick, W. Easson, and C. Greated, "Simulation of wave motion using a lattice gas model,', Int. J. Numer. Methods Fluids 22, 313 (1996).

${ }^{38}$ J. M. Buick, C. A. Greated, and W. J. Easson, "'Investigation of a lattice gas model for surface gravity waves,' Phys. Fluids 9, 2585 (1997).

${ }^{39}$ R. Benzi, S. Succi, and M. Vergassola, "The lattice Boltzmann equation: Theory and application,', Phys. Rep. 222, 145 (1992).

${ }^{40}$ U. Frisch, D. d'Humières, B. Hasslacher, P. Lallemand, Y. Pomeau, and J.-P. Rivet, "Lattice gas hydrodynamics in two and three dimensions,", Complex Syst. 1, 649 (1987).

${ }^{41}$ S. Wolfram, "Cellular automation fluids 1: Basic theory," J. Stat. Phys. 45, 471 (1986).

${ }^{42} \mathrm{X}$. He and L.-S. Luo, "A priori derivation of the lattice Boltzmann equation,'’ Phys. Rev. E 55, 6333 (1997).

${ }^{43}$ U. Frisch, B. Hasslacher, and Y. Pomeau, "Lattice-gas automata for the Navier-Stokes equation,' Phys. Rev. Lett. 56, 1505 (1986)

${ }^{44}$ H. Chen, S. Chen, and W. H. Matthaeus, "Recovery o the Navier-Stokes equation using a lattice-gas Boltzmann method,' Phys. Rev. A 45, 5339 (1992).

${ }^{45}$ R. Cornubert, D. d'Humières, and D. Levermore, “'A Knudsen later theory for lattice gases,', Physica D 47, 241 (1991).

${ }^{46}$ D. P. Ziegler, "Boundary conditions for lattice Boltzmann simulations,", J. Stat. Phys. 71, 1171 (1993).

${ }^{47}$ D. R. Noble, S. Chen, J. G. Georgiadis, and R. O. Buckius, “'A consistent hydrodynamic boundary condition for the lattice Boltzmann method,', Phys. Fluids 7, 203 (1995).

${ }^{48}$ A. K. Gunstensen, D. H. Rothman, S. Zaleski, and G. Zanetti, "Lattice Boltzmann model of immiscible fluid,' Phys. Rev. A 43, 4320 (1991).

${ }^{49}$ E. G. Flekkøy, 'Lattice Bhatnagar-Gross-Krook models for miscible fluids,'” Phys. Rev. E 47, 4247 (1993).

${ }^{50} \mathrm{X}$. Shan and $\mathrm{H}$. Chen, "Lattice Boltzmann model for simulating flows with multiple phases and components,', Phys. Rev. E 47, 1815 (1993).

${ }^{51} \mathrm{X}$. Shan and G. Doolen, "Multi-component lattice-Boltzmann model with interparticle interactions,'” J. Stat. Phys. 81, 379 (1995).

${ }^{52}$ E. Orlandini, M. R. Swift, and J. M. Yeomans, "A lattice Boltzmann model of binary-fluid mixtures,', Europhys. Lett. 32, 463 (1995).
${ }^{53}$ M. R. Swift, E. Orlandini, W. R. Osborn, and J. M. Yeomans, "Lattice Boltzmann simulation of liquid-gas and binary fluid systems,' Phys. Rev. E 54, 5041 (1996).

${ }^{54}$ Routine E04HFF, The NAG Fortran Library, Mark 14, 1990.

${ }^{55}$ P. E. Gill and W. Murry, "Algorithms for the solution of the nonlinear least-squares problem,' SIAM (Soc. Ind. Appl. Math.) J. Numer. Anal. 15, 977 (1978).

${ }^{56}$ J. Lighthill, Waves In Fluids (Cambridge University Press, Cambridge, 1978).

${ }^{57}$ J. S. Turner, Buoyancy Effects in Fluids (Cambridge University Press, Cambridge, 1973).

${ }^{58}$ O. M. Phillips, The Dynamics of the Upper Ocean (Cambridge University Press, Cambridge, 1969).

${ }^{59}$ P. L-F. Liu, "Damping of water waves over porous bed,' ASCE J. Hydraul. Div. 99, 2263 (1973).

${ }^{60}$ R. A. Dalrymple and P. L.-F. Lu, "Waves over soft muds: A two-layer fluid model,' J. Phys. Oceanogr. 8, 1121 (1978).

${ }^{61}$ C. C. Mei and L.-F. Liu, "The damping of surface gravity waves in a bounded liquid,' J. Fluid Mech. 59, 239 (1973).

${ }^{62}$ R. G. Dean and R. A. Dalrymple, Water Wave Mechanics for Engineers and Scientists (Prentice-Hall, Englewood Cliffs, NJ, 1984).

${ }^{63}$ A. Defant, Physical Oceanography (Pergamon Press, New York, 1961), Vol. II.

${ }^{64}$ Routine C05ADF, The NAG Fortran Library, Mark 14, 1990.

${ }^{65}$ D. B. Kothe and W. J. Rider, "Practical considerations for interface tracking methods,', in 6th International Symposium on Computational Fluid Dynamics, Lake Tahoe, Nevada, 4-8 Sept. 1995, edited by M. Hafez, 1995, pp. 638-643.

${ }^{66} \mathrm{~F}$. Wessels and K. Hutter, "Interaction of internal waves with a topographic sill in a two-layered fluid,' J. Phys. Oceanogr. 26, 5 (1996).

${ }^{67}$ S. Diebels, B. Schuster, and K. Hutter, "Nonlinear internal waves over variable topography,' Geophys. Astrophys. Fluid Dyn. 76, 165 (1994).

${ }^{68} \mathrm{X}$. He and G. Doolen, "Lattice Boltzmann method on curvilinear coordinate system: Flow around a circular cyclinder,' J. Comput. Phys. 134, 306 (1997). 